\title{
ASYMPTOTIC FORMULAS FOR PERTURBATIONS IN THE ELECTROMAGNETIC FIELDS DUE TO THE PRESENCE OF INHOMOGENEITIES OF SMALL DIAMETER
}

\author{
Michael S. VOGEliUs ${ }^{1}$ AND DARKO VOLKOV ${ }^{1}$
}

\begin{abstract}
We consider solutions to the time-harmonic Maxwell's Equations of a TE (transverse electric) nature. For such solutions we provide a rigorous derivation of the leading order boundary perturbations resulting from the presence of a finite number of interior inhomogeneities of small diameter. We expect that these formulas will form the basis for very effective computational identification algorithms, aimed at determining information about the inhomogeneities from electromagnetic boundary measurements.
\end{abstract}

Mathematics Subject Classification. 35J25, 35R30, 78A30.

Received: October 19, 1999. Revised: February 11, 2000.

\section{INTRODUCTION}

The homogeneous, time-dependent, linear Maxwell's Equations take the form

$$
\begin{aligned}
\nabla \times \mathbf{E} & =-\mu \frac{\partial}{\partial t} \mathbf{H} \\
\nabla \times \mathbf{H} & =\mathbf{J}_{f}+\epsilon \frac{\partial}{\partial t} \mathbf{E} .
\end{aligned}
$$

$\mathbf{E} \in \mathbb{R}^{3}$, and $\mathbf{H} \in \mathbb{R}^{3}$, is the electric field and the magnetic field respectively. The coefficients $\mu$ and $\epsilon$ are referred to as the magnetic permeability and the electric permittivity of the medium. $\mathbf{J}_{f}$ is the free current - it is related to the field $\mathbf{E}$ by $\mathbf{J}_{f}=\sigma \mathbf{E}$, where $\sigma$ denotes the electric conductivity of the medium. For a discussion of the physical modeling leading to these equations, we refer the reader to [9] or [11].

It is quite common to consider time-harmonic solutions to the above equations, i.e., special solutions of the form

$$
\mathbf{E}(x, t)=\operatorname{Re}\left\{\mathbf{E}(x) \mathrm{e}^{-i \omega t}\right\}, \quad \text { and } \quad \mathbf{H}(x, t)=\operatorname{Re}\left\{\mathbf{H}(x) \mathrm{e}^{-i \omega t}\right\}, \quad x \in \mathbb{R}^{3}, t>0,
$$

where $\omega>0$ denotes the given frequency, and where the complex valued fields $\mathbf{E}(x)$ and $\mathbf{H}(x)$ satisfy

$$
\begin{aligned}
\nabla \times \mathbf{E} & =i \omega \mu \mathbf{H} \\
\nabla \times \mathbf{H} & =(-i \omega \epsilon+\sigma) \mathbf{E} .
\end{aligned}
$$

Keywords and phrases. Maxwell equations, inverse problems.

1 Department of Mathematics, Rutgers University, New Brunswick, NJ 08903, USA. e-mail: vogelius@hilbert.rutgers.edu; dvolkov@math.rutgers. edu 
In order to arrive at particular non-trivial solutions to these equations, it is most natural to either introduce current sources (additive to the free current) or to prescribe non-trivial boundary conditions $(\mathbf{E} \times \nu$ or $\mathbf{H} \times \nu)$ on the boundary of a given domain $\boldsymbol{\Omega}$ ( $\nu$ denotes the outward unit normal to $\boldsymbol{\Omega}$ ). Here we consider only the latter possibility.

An underlying objective of the work described in this paper is to determine, most effectively, properties of the scalar parameters (functions) $\mu>0, \epsilon>0$ and $\sigma \geq 0$ inside $\Omega$ from overdetermined boundary information about specific solutions to (1). In order to put (the objective of) our work in context, let us therefore briefly review the current state of knowledge concerning this "inverse problem", in case the Cauchy boundary data of all solutions are known. The term Cauchy boundary datum is used to denote any pair $\left(\left.\mathbf{E}\right|_{\boldsymbol{\Gamma}} \times \nu,\left.\mathbf{H}\right|_{\boldsymbol{\Gamma}} \times \nu\right) ; \boldsymbol{\Gamma}$ denotes the boundary of $\boldsymbol{\Omega}$. If we assume $\omega$ is not an eigenfrequency, then the set of Cauchy data is the graph of a map, i.e. it consists of pairs of the form $\left(\left.\mathbf{E}\right|_{\boldsymbol{\Gamma}} \times \nu, \Lambda_{\omega}\left(\left.\mathbf{E}\right|_{\boldsymbol{\Gamma}} \times \nu\right)\right)$. The mapping

$$
\Lambda_{\omega}:\left.\mathbf{E}\right|_{\Gamma} \times\left.\nu \rightarrow \mathbf{H}\right|_{\Gamma} \times \nu
$$

is well defined as a mapping from the space $T H_{\text {Div }}^{1 / 2}(\boldsymbol{\Gamma})$ into itself (this latter space is the vector space of $H^{1 / 2}$ vectorfields that are tangential to $\boldsymbol{\Gamma}$, and that have a surface divergence which also lies in $H^{1 / 2}$ ). It is well known that the operator $\Lambda_{\omega}$ (corresponding to just a single positive frequency) uniquely determines the coefficients $\mu, \epsilon$ and $\sigma$, provided these are sufficiently smooth $\left(C^{3}\right)$ and remain so when extended by constants outside $\boldsymbol{\Omega}[16]$. This result is a generalization of an earlier result for the scalar conductivity equation (the case formally corresponding to the eigenfrequency $\omega=0$ ). Indeed in the latter case (so-called direct current) the electric field takes the form of a gradient, $\mathbf{E}=\nabla u$, where the (voltage) potential $u$ now satisfies

$$
\nabla \cdot \sigma \nabla u=0
$$

The tangential field $\left.\mathbf{E}\right|_{\boldsymbol{\Gamma}} \times \nu$ equals $\nabla_{\boldsymbol{\Gamma}} u \times \nu$ (the surface curl of $u$ ). The tangential field $\left.\mathbf{H}\right|_{\boldsymbol{\Gamma}} \times \nu$, on the other side, is only defined modulo a surface curl. This is equivalent to saying that only $\nabla_{\boldsymbol{\Gamma}} \cdot(\mathbf{H} \times \nu)=\operatorname{Div} \boldsymbol{\Gamma}(\mathbf{H} \times \nu)=$ $\left.\nu \cdot(\nabla \times \mathbf{H})\right|_{\boldsymbol{\Gamma}}=\left.\nu \cdot \sigma \mathbf{E}\right|_{\boldsymbol{\Gamma}}=\left.\sigma \frac{\partial u}{\partial \nu}\right|_{\boldsymbol{\Gamma}}$ is well defined. Full knowledge of the map $\Lambda_{0}$ is thus formally equivalent to full knowledge of the Dirichlet to Neumann data map, $\Lambda$, which sends $u_{\Gamma}$ into $\Lambda\left(u_{\Gamma}\right)=\left.\sigma \frac{\partial u}{\partial \nu}\right|_{\Gamma}$. For a rigorous proof of the fact that $\Lambda(f)=\nabla_{\boldsymbol{\Gamma}} \cdot\left(\lim _{\omega \rightarrow 0} \Lambda_{\omega}\right)\left(\nabla_{\boldsymbol{\Gamma}} f \times \nu\right)$, for sufficiently smooth $f$, see [13]. For the conductivity problem it has been known for some time that the Dirichlet to Neumann data map uniquely determines an isotropic conductivity $\sigma$, see $[12,15,20]$.

We now focus on a special case of the three dimensional Maxwell's Equation, namely the case where the coefficients $\mu, \epsilon, \sigma$ and the fields $\mathbf{E}, \mathbf{H}$ are independent of one of the variables, say $x_{3}$ (and the domain takes the form of a cylinder parallel to the $x_{3}$-axis). In this case the Maxwell's Equations split into two sets of independent equations, one for the fields $\mathbf{E}^{*}=\left(0,0, E_{3}\right), \mathbf{H}^{*}=\left(H_{1}, H_{2}, 0\right)$ and one for the fields $\mathbf{E}^{* *}=\left(E_{1}, E_{2}, 0\right), \mathbf{H}^{* *}=\left(0,0, H_{3}\right)$. The first set of equations are associated with the terminology TE (transverse electric), the second set with the terminology TM (transverse magnetic). We note that the (full) Maxwell's Equations can be reformulated as an equation for the electric field

$$
\nabla \times\left(\frac{1}{\mu} \nabla \times \mathbf{E}\right)-\omega^{2}\left(\epsilon+i \frac{\sigma}{\omega}\right) \mathbf{E}=0
$$

with the magnetic field given by

$$
\mathbf{H}=\frac{1}{i \omega \mu} \nabla \times \mathbf{E}
$$




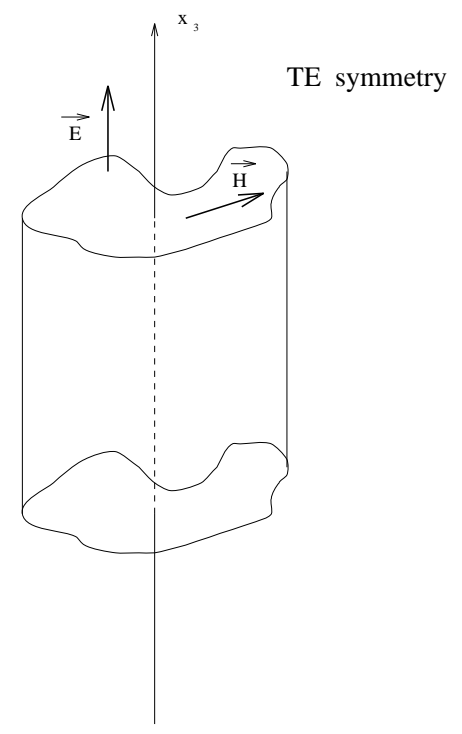

Figure 1.

Consider now the TE situation. Let $\Omega$ denote the cross section of the (vertical) cylinder, and let $\tilde{x}$ denote the "cross sectional coordinates" $\tilde{x}=\left(x_{1}, x_{2}\right)$. The equation for $\mathbf{E}^{*}$ transforms into the following scalar equation

$$
\nabla_{\tilde{x}} \cdot\left(\frac{1}{\mu} \nabla_{\tilde{x}} E_{3}\right)+\omega^{2}\left(\epsilon+i \frac{\sigma}{\omega}\right) E_{3}=0
$$

for $E_{3}$, with the corresponding magnetic field $\mathbf{H}^{*}$ given by

$$
\mathbf{H}^{*}=\frac{1}{i \omega \mu}\left(\frac{\partial}{\partial x_{2}} E_{3},-\frac{\partial}{\partial x_{1}} E_{3}, 0\right)
$$

Knowing $\mathbf{E}^{*} \times \nu$ on the (vertical) boundary of the three dimensional domain amounts to knowing $E_{3}$ on the boundary of the two dimensional cross section. Knowing $\mathbf{H}^{*} \times \nu$ on the (vertical) boundary of the three dimensional domain amounts to knowing $\frac{1}{\mu} \frac{\partial}{\partial \nu} E_{3}$ on the boundary of the two dimensional cross section. Knowledge of the boundary mapping $\mathbf{E}^{*} \times \nu \rightarrow \mathbf{H}^{*} \times \nu$ is thus equivalent to knowledge of the Dirichlet to Neumann map $\Lambda_{\omega}^{*}:\left.\left.E_{3}\right|_{\partial \Omega} \rightarrow \frac{1}{\mu} \frac{\partial}{\partial \nu} E_{3}\right|_{\partial \Omega}$. If $\mu, \epsilon$ and $\sigma$ are sufficiently smooth and sufficiently close to constant, then one may use the arguments in [10] to show that full knowledge of $\Lambda_{\omega}^{*}$ for two different frequencies, $\omega_{1}$ and $\omega_{2}$, is sufficient to determine these three scalar coefficients. The argument roughly goes as follows: $\Lambda_{\omega}^{*}$ determines $\frac{1}{\mu}$ as well as $\frac{\partial}{\partial \nu}\left(\frac{1}{\mu}\right)$ on the boundary; by introducing $v=\left(\sqrt{\frac{1}{\mu}}\right) E_{3}$, the identification problem now becomes equivalent to determining $\mu, \epsilon$ and $\sigma$ from knowledge of the map $\Phi_{\omega}:\left.\left.v\right|_{\partial \Omega} \rightarrow \frac{\partial v}{\partial \nu}\right|_{\partial \Omega}$, where $v$ is any solution to the equation

$$
\triangle v+\left(\omega^{2} \epsilon \mu+i \omega \sigma \mu-\sqrt{\mu} \triangle\left(\sqrt{\frac{1}{\mu}}\right)\right) v=0 .
$$

If we suppose $\mu, \epsilon$ and $\sigma$ are sufficiently smooth and close to constant, then knowledge of $\Phi_{\omega_{1}}$ (for a single value of $\omega$ ) allows the unique determination of $\sigma \mu$ and $\omega_{1}^{2} \epsilon \mu-\sqrt{\mu} \triangle\left(\sqrt{\frac{1}{\mu}}\right)$ (a simple extension of the core result in [19] to complex potentials). However, this single-frequency knowledge is clearly insufficient for the determination of the individual functions $\mu, \epsilon$ and $\sigma$. The requirement, that the functions be close to constant, owes to the 
fact that we are in two dimension. If we additionally know $\Phi_{\omega_{2}}$, for a frequency $\omega_{2} \neq \omega_{1}$, then we immediately arrive at knowledge of $\sigma \mu, \epsilon \mu$ and $\sqrt{\mu} \triangle\left(\sqrt{\frac{1}{\mu}}\right)$. Since $\frac{1}{\mu}$ and $\frac{\partial}{\partial \nu}\left(\frac{1}{\mu}\right)$ are known on the boundary this allows the determination of $\mu$ (due to the uniqueness of the solution to the corresponding Cauchy problem for $\sqrt{\frac{1}{\mu}}$ ). Finally we may now also determine $\epsilon$ and $\sigma$.

The aim of this paper is to derive asymptotic formulas for the electric and magnetic fields in the practically very interesting situation, where a number of objects of small diameter and with different material characteristics are imbedded in an otherwise smooth medium. For simplicity we assume that the material characteristics of each of the inhomogeneities as well as those of the background medium are constant. This simplification allows us to base our analysis largely on boundary integral methods; it also allows us to use an explicit fundamental solution for the underlying Helmholtz Equation. In view of the above identification results, and based on our experience with the direct current conductivity problem, we expect that (the boundary traces of) our formulas will allow very effective determination of the location and the size of the imbedded objects. It should be particularly interesting to derive numerical reconstruction algorithms based on these formulas and on principles, similar to those described in $[1,3,5,17]$. A particularly challenging practical application would be the determination of the location of antipersonnel- and other types of mines. Another application would be to so-called "eddy current methods", which are now frequently used for corrosion- and other metal defect inspection $(c f .[4,7])$.

In this paper we concentrate on the situation of TE symmetry. Entirely similar formulas could be derived in the case of TM symmetry. It is the focus of current work to rigorously derive corresponding formulas for the solutions to the (full) three dimensional Maxwell's Equations. Although the formulas we derive in this paper may be regarded as generalizations of those already derived in [5], the analysis presented here is quite different and entirely selfcontained.

\section{The MAIN RESUlts}

Let $\Omega$ be a bounded, smooth subdomain of $\mathbb{R}^{2}$. For simplicity we take $\partial \Omega$ to be $C^{\infty}$, but this condition could be considerably weakened. We suppose that $\Omega$ contains a finite number of inhomogeneities, each of the form $z_{j}+\rho B_{j}$, where $B_{j} \subset \mathbb{R}^{2}$ is a bounded, smooth $\left(C^{\infty}\right)$ domain containing the origin. The total collection of inhomogeneities thus takes the form $\mathcal{I}_{\rho}=\cup_{j=1}^{m}\left(z_{j}+\rho B_{j}\right)$. The points $z_{j} \in \Omega, j=1, \ldots$, $m$, that determine the location of the inhomogeneities are assumed to satisfy

$$
\begin{aligned}
& 0<d_{0} \leq\left|z_{j}-z_{l}\right| \quad \forall j \neq l \\
& 0<d_{0} \leq \operatorname{dist}\left(z_{j}, \partial \Omega\right) \quad \forall j
\end{aligned}
$$

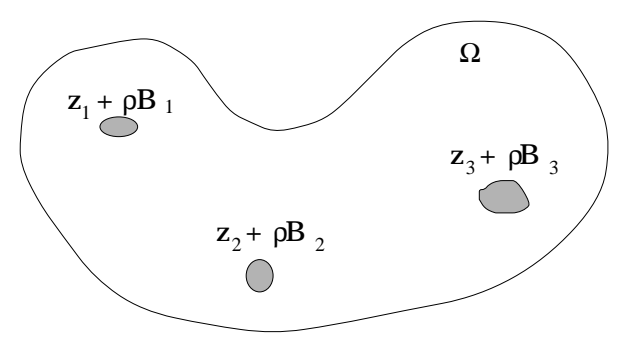

Figure 2. 
As a consequence of this assumption it follows immediately that

$$
m \leq 4|\Omega| / \pi d_{0}^{2}
$$

We also assume that $\rho>0$, the common order of magnitude of the diameters of the inhomogeneities, is sufficiently small that these are disjoint and that their distance to $\mathbb{R}^{2} \backslash \Omega$ is larger than $d_{0} / 2$. Let $\mu^{0}>0$, $\epsilon^{0}>0$ and $\sigma^{0} \geq 0$ denote the permeability, the permittivity and the conductivity of the background medium; for simplicity we shall in this paper assume that these are constants. Let $\mu^{j}>0, \epsilon^{j}>0$ and $\sigma^{j} \geq 0$ denote the constant permeability, permittivity and conductivity of the $j$ th inhomogeneity, $z_{j}+\rho B_{j}$. Using this notation we introduce the piecewise constant magnetic permeability

$$
\mu_{\rho}(x)= \begin{cases}\mu^{0}, & x \in \Omega \backslash \overline{\mathcal{I}}_{\rho} \\ \mu^{j}, & x \in z_{j}+\rho B_{j}, j=1 \ldots m .\end{cases}
$$

The piecewise constant electric permittivity and electric conductivity are defined analogously. $\omega>0$ is the given frequency. The electric field (or rather, the transversal strength) in the presence of the inhomogeneities, is denoted $E_{\rho}$. It is the solution to

$$
\nabla \cdot\left(\frac{1}{\mu_{\rho}} \nabla E_{\rho}\right)+\omega^{2}\left(\epsilon_{\rho}+i \frac{\sigma_{\rho}}{\omega}\right) E_{\rho}=0, \quad \text { in } \Omega,
$$

with

$$
E_{\rho}=f, \quad \text { on } \partial \Omega
$$

The equation (4) may alternatively be formulated as follows

$$
\begin{aligned}
& \nabla \cdot\left(\frac{1}{\mu^{0}} \nabla E_{\rho}\right)+\omega^{2}\left(\epsilon^{0}+i \frac{\sigma^{0}}{\omega}\right) E_{\rho}=0 \quad \text { in } \Omega \backslash \overline{\mathcal{I}}_{\rho} \\
& \nabla \cdot\left(\frac{1}{\mu^{j}} \nabla E_{\rho}\right)+\omega^{2}\left(\epsilon^{j}+i \frac{\sigma^{j}}{\omega}\right) E_{\rho}=0 \quad \text { in } z_{j}+\rho B_{j} \\
& E_{\rho}^{+}=E_{\rho}^{-} \quad \text { and } \frac{1}{\mu^{0}}\left(\nabla E_{\rho} \cdot \nu\right)^{+}=\frac{1}{\mu^{j}}\left(\nabla E_{\rho} \cdot \nu\right)^{-} \quad \text { on } \partial\left(z_{j}+\rho B_{j}\right) .
\end{aligned}
$$

Here $\nu$ denotes the outward unit normal to $\partial\left(z_{j}+\rho B_{j}\right)$; superscript + and - indicate the limiting values as we approach $\partial\left(z_{j}+\rho B_{j}\right)$ from outside $z_{j}+\rho B_{j}$, and from inside $z_{j}+\rho B_{j}$, respectively. The electric field, $E$, in the absence of any inhomogeneities, satisfies

$$
\nabla \cdot\left(\frac{1}{\mu^{0}} \nabla E\right)+\omega^{2}\left(\epsilon^{0}+i \frac{\sigma^{0}}{\omega}\right) E=0 \quad \text { in } \Omega,
$$

with

$$
E=f \quad \text { on } \partial \Omega
$$

Since all the involved coefficients are constants, this may also be rewritten

$$
\left(\Delta+k^{2}\right) E=0 \quad \text { in } \Omega \text {, with } E=f \quad \text { on } \partial \Omega .
$$

Here $k^{2}$ is the (complex) constant

$$
k^{2}=\omega^{2} \mu^{0}\left(\epsilon^{0}+i \frac{\sigma^{0}}{\omega}\right) .
$$


In order to insure well-posedness (also for the $\rho$ dependent problem) we shall always assume that

$$
-k^{2} \text { is not an eigenvalue for the operator }
$$

$\Delta$ with Dirichlet boundary conditions.

Before formulating the main results of this paper we need to introduce some additional notation. For any $1 \leq j \leq m$, let $\phi_{j}$ denote the vector valued solution to

$$
\left\{\begin{array}{l}
\Delta \phi_{j}=0 \text { in } B_{j}, \text { and in } \mathbb{R}^{2} \backslash \overline{B_{j}} \\
\phi_{j} \text { is continuous across } \partial B_{j} \\
\frac{\mu^{j}}{\mu^{0}}\left(\frac{\partial \phi_{j}}{\partial \nu}\right)^{+}-\left(\frac{\partial \phi_{j}}{\partial \nu}\right)^{-}=-\nu \\
\lim _{|z| \rightarrow \infty}\left|\phi_{j}(z)\right|=0 .
\end{array}\right.
$$

The existence and uniqueness of this $\phi_{j}$ can be established using single layer potentials with suitably chosen densities, see [5]. We note that for a fixed $j, \phi_{j}=\phi_{1}^{j} e_{1}+\phi_{2}^{j} e_{2}$, where $\left\{e_{1}, e_{2}\right\}$ is the standard basis for $\mathbb{R}^{2}$, and $\phi_{1}^{j}, \phi_{2}^{j}$ are the scalar functions introduced in [5].

The polarization tensor, $M_{j}$, corresponding to the $j$ 'th inhomogeneity is now given by

$$
M_{j}\left(\frac{\mu^{j}}{\mu^{0}}\right)=\left|B_{j}\right| I+\left(\frac{\mu^{j}}{\mu^{0}}-1\right) \int_{\partial B_{j}} \nu(y)\left(\phi_{j}(y)\right)^{T} \mathrm{~d} s(y) .
$$

We have used the notation $M_{j}\left(\frac{\mu^{j}}{\mu^{0}}\right)$ to signify that, for a fixed shape of the inhomogeneity $B_{j}$, the polarization tensor depends only on the ratio $\frac{\mu^{j}}{\mu^{0}}$. It is not difficult to prove that $M_{j}$ is a symmetric, positive definite $2 \times 2$ matrix $\left(M_{j}\right.$ is up to a factor of $\frac{\mu^{j}}{\mu^{0}}$ identical to the rescaled polarization tensor introduced in [5]).

Let $H_{0}^{(1)}$ denote the Hankel function of the first kind of order zero (sometimes this is also referred to as a Bessel function of the third kind), see p. 108 in [14] or p. 73 in [21]. We introduce the function

$$
\Phi^{k}(x, y)=\frac{i}{4} H_{0}^{(1)}(k|x-y|)
$$

We recall that for $w$ a non zero complex number with $-\pi<\operatorname{Arg} w<\pi, H_{0}^{(1)}(w)$ is given by

$$
H_{0}^{(1)}(w)=J_{0}(w)+i Y_{0}(w)
$$

where $J_{0}$ is the Bessel function of the first kind of order 0 :

$$
J_{0}(w)=\sum_{m=0}^{\infty} \frac{(-1)^{m}}{(m !)^{2}}\left(\frac{1}{2} w\right)^{2 m}
$$

and $Y_{0}$ is the Weber-Schläfli function (sometimes also referred to as a Bessel function of the second kind) of order 0:

$$
Y_{0}(w)=\frac{2}{\pi}\left\{\left(\gamma+\log \left(\frac{1}{2} w\right)\right) J_{0}(w)-\sum_{m=1}^{\infty} \frac{(-1)^{m}}{(m !)^{2}}\left(\frac{1}{2} w\right)^{2 m}\left(\frac{1}{1}+\frac{1}{2}+\cdots+\frac{1}{m}\right)\right\}
$$

Here $\gamma$ denotes Euler's constant

$$
\gamma=\lim _{m \rightarrow \infty}\left\{\left(\frac{1}{1}+\frac{1}{2}+\cdots+\frac{1}{m}\right)-\log m\right\}
$$


For more details we refer to Chapter 3 in [21]. The function $\Phi^{k}$ is a "free space" Green's function for $\Delta+k^{2}$, in other words, it satisfies

$$
\left(\Delta+k^{2}\right) \Phi^{k}(\cdot, y)=-\delta_{y} \quad \text { in } \mathbb{R}^{2}
$$

One of the main results proven in this paper is the following asymptotic formula concerning the perturbation, $\left.\left(\frac{\partial E_{\rho}}{\partial \nu}-\frac{\partial E}{\partial \nu}\right)\right|_{\partial \Omega}$, in the (rescaled) boundary magnetic field, caused by the presence of the inhomogeneities. In this connection we note that, even though the individual normal derivatives $\left(\frac{\partial E_{\rho}}{\partial \nu}\right.$ and $\left.\frac{\partial E}{\partial \nu}\right)$ may only be defined in a distributional sense on $\partial \Omega$ (if $f$ is just in $H^{1 / 2}(\partial \Omega)$ ) elliptic regularity results ensure that the perturbation $\left.\left(\frac{\partial E_{\rho}}{\partial \nu}-\frac{\partial E}{\partial \nu}\right)\right|_{\partial \Omega}$ is indeed an infinitely smooth function.

Theorem 1. Suppose (2) and (8) are satisfied. There exists $0<\rho_{0}$ such that, given an arbitrary $f \in H^{1 / 2}(\partial \Omega)$, and any $0<\rho<\rho_{0}$, the boundary value problem (4)-(5) has a unique solution $E_{\rho}$. The constant $\rho_{0}$ depends on the domains $\left\{B_{j}\right\}_{j=1}^{m}, \Omega$, the constants $\left\{\mu^{j}, \epsilon^{j}, \sigma^{j}\right\}_{j=0}^{m}$, the frequency $\omega$, and $d_{0}$, but is otherwise independent of the points $\left\{z_{j}\right\}_{j=1}^{m}$. Let $E$ denote the unique solution to the boundary value problem (6)-(7) corresponding to the same $f \in H^{1 / 2}(\partial \Omega)$. For any $x \in \partial \Omega$ we then have

$$
\begin{aligned}
& \frac{\partial E_{\rho}}{\partial \nu}(x)-\frac{\partial E}{\partial \nu}(x)-2 \int_{\partial \Omega}\left(\frac{\partial E_{\rho}}{\partial \nu}-\frac{\partial E}{\partial \nu}\right)(y) \frac{\partial \Phi^{k}(x, y)}{\partial \nu(x)} \mathrm{d} s(y) \\
& =2 \rho^{2} \sum_{j=1}^{m}\left(1-\frac{\mu^{0}}{\mu^{j}}\right) \nabla_{y} \frac{\partial \Phi^{k}}{\partial \nu(x)}\left(x, z_{j}\right) \cdot M_{j}\left(\frac{\mu^{j}}{\mu^{0}}\right) \nabla E\left(z_{j}\right) \\
& -2 \rho^{2} \omega^{2} \mu^{0} \sum_{j=1}^{m}\left(\epsilon^{0}-\epsilon^{j}+i \frac{\sigma^{0}-\sigma^{j}}{\omega}\right) \frac{\partial \Phi^{k}}{\partial \nu(x)}\left(x, z_{j}\right)\left|B_{j}\right| E\left(z_{j}\right)+O\left(\rho^{3-\delta}\right) .
\end{aligned}
$$

For any fixed $\delta>0$, the term $O\left(\rho^{3-\delta}\right)$ is bounded by $C \rho^{3-\delta}$, uniformly in $x$. The constant $C$ depends on $\delta$, the domains $\left\{B_{j}\right\}_{j=1}^{m}, \Omega$, the constants $\left\{\mu^{j}, \epsilon^{j}, \sigma^{j}\right\}_{j=0}^{m}$, the frequency $\omega,\|f\|_{H^{1 / 2}(\partial \Omega)}$, and $d_{0}$, but is otherwise independent of the points $\left\{z_{j}\right\}_{j=1}^{m}$.

Let $\tilde{E}_{\rho}$ and $\tilde{E}$ denote solutions to the same equations as $E_{\rho}$ and $E$, but with Neumann- instead of Dirichlet boundary conditions, i.e.,

$$
\frac{1}{\mu^{0}} \frac{\partial \tilde{E}_{\rho}}{\partial \nu}=\frac{1}{\mu^{0}} \frac{\partial \tilde{E}}{\partial \nu}=g \quad \text { on } \partial \Omega
$$

We also derive an asymptotic formula concerning the perturbation $\tilde{E}_{\rho}-\tilde{E}$.

Theorem 2. Suppose that $-k^{2}=-\omega^{2} \mu^{0}\left(\epsilon^{0}+i \frac{\sigma^{0}}{\omega}\right)$ is not an eigenvalue for the Laplacian with Neumann boundary conditions, and suppose that (2) is satisfied. There exists $0<\rho_{0}$ such that, given any $g \in H^{-1 / 2}(\partial \Omega)$, and any $0<\rho<\rho_{0}$, the boundary value problem (4), (11) has a unique solution $\tilde{E}_{\rho}$. The constant $\rho_{0}$ depends on the domains $\left\{B_{j}\right\}_{j=1}^{m}, \Omega$, the constants $\left\{\mu^{j}, \epsilon^{j}, \sigma^{j}\right\}_{j=0}^{m}$, the frequency $\omega$, and $d_{0}$, but is otherwise independent of the points $\left\{z_{j}\right\}_{j=1}^{m}$. Let $\tilde{E}$ denote the unique solution to the boundary value problem (6), (11) corresponding 
to the same $g \in H^{-1 / 2}(\partial \Omega)$. For any $x \in \partial \Omega$ we then have

$$
\begin{aligned}
& \tilde{E}_{\rho}(x)-\tilde{E}(x)+2 \int_{\partial \Omega}\left(\tilde{E}_{\rho}-\tilde{E}\right)(y) \frac{\partial \Phi^{k}(x, y)}{\partial \nu(y)} \mathrm{d} s(y) \\
&=2 \rho^{2} \sum_{j=1}^{m}\left(1-\frac{\mu^{0}}{\mu^{j}}\right) \nabla_{y} \Phi^{k}\left(x, z_{j}\right) \cdot M_{j}\left(\frac{\mu^{j}}{\mu^{0}}\right) \nabla \tilde{E}\left(z_{j}\right) \\
&\left.\quad-2 \rho^{2} \omega^{2} \mu^{0} \sum_{j=1}^{m}\left(\epsilon^{0}-\epsilon^{j}+i \frac{\sigma^{0}-\sigma^{j}}{\omega}\right) \Phi^{k}\left(x, z_{j}\right)\right)\left|B_{j}\right| \tilde{E}\left(z_{j}\right)+O\left(\rho^{3-\delta}\right) .
\end{aligned}
$$

For any fixed $\delta>0$, the term $O\left(\rho^{3-\delta}\right)$ is bounded by $C \rho^{3-\delta}$, uniformly in $x$. The constant $C$ depends on $\delta$, the domains $\left\{B_{j}\right\}_{j=1}^{m}, \Omega$, the constants $\left\{\mu^{j}, \epsilon^{j}, \sigma^{j}\right\}_{j=0}^{m}$, the frequency $\omega,\|g\|_{H^{-1 / 2}(\partial \Omega)}$, and $d_{0}$, but is otherwise independent of the points $\left\{z_{j}\right\}_{j=1}^{m}$.

We notice that the asymptotic formula in this last theorem represents a generalization of that stated in Remark 2 of [5], where we considered "only" the direct current conductivity problem. We also notice that Theorem 1 and Theorem 2 (as well as the proofs we provide) have direct analogs for dimension higher than 2 however in that case the equations satisfied by $E_{\rho}\left(\tilde{E}_{\rho}\right)$ and $E(\tilde{E})$ have no relation to the Maxwell's Equations - and so we have decided not to include that "generalization" here.

The next four sections are devoted to a proof of Theorem 1. Briefly described the proof proceeds as follows. In Section 3 we establish the well-posedness of the problem (4)-(5); to be more specific, we use the theory of collectively compact operators to prove that if the situation corresponding to $\rho=0$ (the background situation) is not at an eigenfrequency, then the boundary value problem (4)-(5), for $\rho$ sufficiently small, has a unique solution, which is bounded independently of $\rho$. As a first step we then derive, in Section 4, an energy estimate for the difference $E_{\rho}-E$. Based on this estimate and a boundary integral formulation we are now able to obtain an asymptotic formula for $E_{\rho}-E$ on the boundary of each of the inhomogeneities. This formula is found in Proposition 3 of Section 5. By a fairly straightforward application of Green's formula (as detailed at the beginning of Sect. 6) the values of $\left(E_{\rho}-E\right)_{\partial \Omega}$ plus a certain convolution of the corresponding normal derivative can be related to a combination of appropriate integrals of $E_{\rho}$ over the inhomogeneities and over their boundaries. The formula from Proposition 3 now allows us to express those integrals in terms of the polarization tensors, the volumes of the inhomogeneities, the Hankel function and the background field $E$. This all leads to the proof of Theorem 1, given at the end Section 6. As a final remark there is a brief outline of the (rather minimal) changes required for a proof of Theorem 2. In Sections 5 and 6 we restrict, for simplicity, our attention to the case of a single inhomogeneity, i.e., the case $m=1$.

\section{WELL-POSEDNESS}

From the assumption (8) it follows immediately that the constant coefficient problem

$$
\begin{cases}\nabla \cdot\left(\frac{1}{\mu^{0}} \nabla u\right)+\omega^{2}\left(\epsilon^{0}+i \frac{\sigma^{0}}{\omega}\right) u=F & \text { in } \Omega \\ u=0 & \text { on } \partial \Omega\end{cases}
$$

is well-posed: for any $F \in H^{-1}(\Omega)$ this problem always has a unique (variational) solution, and furthermore there exists a constant $C$ such that $\|u\|_{H^{1}(\Omega)} \leq C\|F\|_{H^{-1}(\Omega)}$. In this Section we prove that the assumption (8) also guarantees that the problem

$$
\begin{cases}\nabla \cdot\left(\frac{1}{\mu_{\rho}} \nabla u_{\rho}\right)+\omega^{2}\left(\epsilon_{\rho}+i \frac{\sigma_{\rho}}{\omega}\right) u_{\rho}=F & \text { in } \Omega \\ u_{\rho}=0 & \text { on } \partial \Omega\end{cases}
$$

is well-posed for $\rho$ sufficiently small. Here the piecewise constant coefficients $\mu_{\rho}, \epsilon_{\rho}$ and $\sigma_{\rho}$ are as defined in (3). 
Proposition 1. Suppose (2) and (8) are satisfied. There exist constants $0<\rho_{0}$ and $C$ such that, given any $0<\rho<\rho_{0}$ and any $F \in H^{-1}(\Omega)$, the problem (12) has a unique (variational) solution, $u_{\rho} \in H_{0}^{1}(\Omega)$. This solution furthermore satisfies

$$
\left\|u_{\rho}\right\|_{H^{1}(\Omega)} \leq C\|F\|_{H^{-1}(\Omega)} .
$$

The constants $\rho_{0}$ and $C$ depend on the domains $\left\{B_{j}\right\}_{j=1}^{m}$, the domain $\Omega$, the constants $\left\{\mu^{j}, \epsilon^{j}, \sigma^{j}\right\}_{j=0}^{m}$, the frequency $\omega$, and $d_{0}$, but is otherwise independent of the points $\left\{z_{j}\right\}_{j=1}^{m}$.

It follows as an immediate corollary that:

Corollary 1. Suppose (2) and (8) are satisfied. There exist constants $0<\rho_{0}$ and $C$ such that, given any $0<\rho<\rho_{0}$ and any $f \in H^{1 / 2}(\partial \Omega)$, the problem (4)-(5) has a unique (variational) solution, $E_{\rho} \in H^{1}(\Omega)$. This solution furthermore satisfies

$$
\left\|E_{\rho}\right\|_{H^{1}(\Omega)} \leq C\|f\|_{H^{1 / 2}(\partial \Omega)} .
$$

The constants $\rho_{0}$ and $C$ depend on the domains $\left\{B_{j}\right\}_{j=1}^{m}$, the domain $\Omega$, the constants $\left\{\mu^{j}, \epsilon^{j}, \sigma^{j}\right\}_{j=0}^{m}$, the frequency $\omega$, and $d_{0}$, but is otherwise independent of the points $\left\{z_{j}\right\}_{j=1}^{m}$.

In order to prove Proposition 1 it is useful to make two observations concerning convergent series of bounded linear operators. The first observation is:

Lemma 1. Let $T, T_{n}, n=1,2, \cdots \quad$ be bounded linear operators mapping one Banach space $\mathcal{B}_{1}$ onto another Banach space $\mathcal{B}_{2}$. Suppose that $T_{n} \rightarrow T$, pointwise. If $T_{n}^{-1}$ is well defined and $\left\|T_{n}^{-1}\right\|_{\mathcal{B} \mathcal{L}\left(\mathcal{B}_{2}, \mathcal{B}_{1}\right)}$ is bounded independently of $n$, then $T^{-1}$ exists, and $T_{n}^{-1} \rightarrow T^{-1}$, pointwise.

Proof. Since

$$
c\|x\|_{1} \leq\left\|T_{n} x\right\|_{2},
$$

it follows immediately from the assumption about pointwise convergence, that

$$
c\|x\|_{1} \leq\|T x\|_{2}
$$

and hence, that $T^{-1}$ is well defined. Since

$$
T_{n} T^{-1} y \rightarrow T T^{-1} y=y, \quad \text { as } n \rightarrow \infty
$$

we obtain

$$
c\left\|T_{n}^{-1} y-T^{-1} y\right\|_{1} \leq\left\|y-T_{n} T^{-1} y\right\|_{2} \rightarrow 0, \quad \text { as } n \rightarrow \infty .
$$

This ensures the pointwise convergence of $T_{n}^{-1}$ to $T^{-1}$.

For the second observation we need the notion of collective compactness. Let $\left\{T_{n}\right\}_{n=1}^{\infty}$ be a sequence of bounded, linear operators of a Banach space B (into itself). We shall say that $\left\{T_{n}\right\}_{n=1}^{\infty}$ is collectively compact iff the set $\left\{T_{n}(x): 1 \leq n,\|x\| \leq 1\right\}$ is relatively compact (i.e. its closure is compact).

Lemma 2. Let $T, T_{n}, n=1,2, \cdots \quad$ be bounded, linear operators of a Banach space B. Assume that $T_{n} \rightarrow T$, pointwise, and that $\left\{T_{n}-T\right\}_{n=1}^{\infty}$ is collectively compact. For any scalar, $\lambda$, the two following statements are equivalent:

(a) $\lambda-T$ is an isomorphism;

(b) there exists $N$ such that $\lambda-T_{n}$ is an isomorphism for $n \geq N$, and the set $\left\{\left(\lambda-T_{n}\right)^{-1}: n \geq N\right\}$ is norm bounded. 
Proof. This is the first assertion of Theorem 4.3 in [2]. We refer the reader to that book for a proof.

It is convenient to introduce the constants

$$
\gamma^{j}=\frac{1}{\mu^{j}}, \quad q^{j}=\omega^{2}\left(\epsilon^{j}+i \frac{\sigma^{j}}{\omega}\right)
$$

and the corresponding functions

$$
\gamma_{\rho}=\frac{1}{\mu_{\rho}}, \quad q_{\rho}=\omega^{2}\left(\epsilon_{\rho}+i \frac{\sigma_{\rho}}{\omega}\right) .
$$

For a fixed positive constant, $s$, with

$$
s \geq \max _{0 \leq j \leq m}\left\{\operatorname{Re} q^{j}\right\}
$$

let $L_{\rho,\left\{z_{j}\right\}}: H_{0}^{1}(\Omega) \rightarrow H^{-1}(\Omega)$ denote the linear operator defined by

$$
L_{\rho,\left\{z_{j}\right\}} u=\nabla \cdot\left(\gamma_{\rho} \nabla u\right)+q_{\rho} u-s u
$$

Given $u$ and $v$ in $H_{0}^{1}(\Omega)$

$$
\begin{aligned}
\left|\left\langle L_{\rho,\left\{z_{j}\right\}} u, v\right\rangle\right| & =\left|\int_{\Omega} \gamma_{\rho} \nabla u \overline{\nabla v} \mathrm{~d} x-\int_{\Omega} q_{\rho} u \bar{v} \mathrm{~d} x+s \int_{\Omega} u \bar{v} \mathrm{~d} x\right| \\
& \leq C\|u\|_{H^{1}(\Omega)}\|v\|_{H^{1}(\Omega)}
\end{aligned}
$$

where $\langle$,$\rangle represents the usual duality pairing between H_{0}^{1}(\Omega)$ and $H^{-1}(\Omega)$ (the natural extension of the $L^{2}$ inner-product). Due to the lower bound for $s$, we also have

$$
\begin{aligned}
\left|\left\langle L_{\rho,\left\{z_{j}\right\}} u, u\right\rangle\right| & =\left.\left|\int_{\Omega} \gamma_{\rho}\right| \nabla u\right|^{2} \mathrm{~d} x-\int_{\Omega} q_{\rho}|u|^{2} \mathrm{~d} x+s \int_{\Omega}|u|^{2} \mathrm{~d} x \mid \\
& \geq c \int_{\Omega}|\nabla u|^{2} \mathrm{~d} x \geq c\left(\int_{\Omega}|\nabla u|^{2} \mathrm{~d} x+\int_{\Omega}|u|^{2} \mathrm{~d} x\right) .
\end{aligned}
$$

These two estimates show that $L_{\rho,\left\{z_{j}\right\}}$ is continuous and invertible, and that there are positive two constants $C_{1}$ and $C_{2}$, independent of $\rho$ and $\left\{z_{j}\right\}_{j=1}^{m}$, such that

$$
\left\|L_{\rho,\left\{z_{j}\right\}}\right\|_{\mathcal{B} \mathcal{L}\left(H_{0}^{1}(\Omega), H^{-1}(\Omega)\right)} \leq C_{1}, \quad \text { and } \quad\left\|\left(L_{\rho,\left\{z_{j}\right\}}\right)^{-1}\right\|_{\mathcal{B L}\left(H^{-1}(\Omega), H_{0}^{1}(\Omega)\right)} \leq C_{2} .
$$

Let $F$ be in $H^{-1}(\Omega)$; the equation (12) is equivalent to

$$
\nabla \cdot\left(\gamma_{\rho} \nabla u\right)+q_{\rho} u=F,
$$

with $u \in H_{0}^{1}(\Omega)$. This latter equation may be rewritten

$$
L_{\rho,\left\{z_{j}\right\}} u+s I u=F,
$$

where $I$ denotes the natural compact injection $H_{0}^{1}(\Omega) \rightarrow H^{-1}(\Omega)$. Finally, (16) is equivalent to

$$
u+s\left(L_{\rho,\left\{z_{j}\right\}}\right)^{-1} I u=\left(L_{\rho,\left\{z_{j}\right\}}\right)^{-1} F .
$$


Lemma 3. Let $s$ be a positive constant satisfying (13). Let $\rho_{n}$ be a sequence with $\rho_{n} \rightarrow 0$, as $n \rightarrow \infty$, and let $\left\{z_{j}^{n}\right\}_{j=1}^{m}$ be a sequence of sets of points in $\Omega$ satisfying (2). The sequence of operators $\left(L_{\rho_{n},\left\{z_{j}^{n}\right\}}\right)^{-1} I \quad($ from $H_{0}^{1}(\Omega)$ to itself) is collectively compact and converges pointwise to $L_{0}^{-1} I$, where $L_{0}: H_{0}^{1}(\Omega) \rightarrow H^{-1}(\Omega)$ is defined by

$$
L_{0} u=\nabla \cdot\left(\gamma^{0} \nabla u\right)+q^{0} u-s u \text {. }
$$

Proof. To verify the pointwise convergence, we observe that for $u$ and $v$ in $H_{0}^{1}(\Omega)$

$$
\begin{aligned}
\left|\left\langle\left(L_{\rho_{n},\left\{z_{j}^{n}\right\}}-L_{0}\right) u, v\right\rangle\right| & =\left|-\int_{\mathcal{I}_{\rho_{n}}}\left(\gamma_{\rho_{n}}-\gamma^{0}\right) \nabla u \overline{\nabla v} \mathrm{~d} x+\int_{\mathcal{I}_{\rho_{n}}}\left(q_{\rho_{n}}-q^{0}\right) u \bar{v} \mathrm{~d} x\right| \\
& \leq C\|v\|_{H^{1}(\Omega)}\left(\|\nabla u\|_{L^{2}\left(\mathcal{I}_{\rho_{n}}\right)}+\|u\|_{L^{2}\left(\mathcal{I}_{\rho_{n}}\right)}\right) .
\end{aligned}
$$

Since $\left|\mathcal{I}_{\rho_{n}}\right|=\left|\cup_{j=1}^{m}\left(z_{j}^{n}+\rho_{n} B_{j}\right)\right| \rightarrow 0$ as $n \rightarrow \infty$, this proves that $\left(L_{\rho_{n},\left\{z_{j}^{n}\right\}}-L_{0}\right) u \rightarrow 0$ in $H^{-1}(\Omega)$, or in other words, that $L_{\rho_{n},\left\{z_{j}^{n}\right\}}$ converge pointwise to $L_{0}$. Since the operators $L_{\rho_{n},\left\{z_{j}^{n}\right\}}$ (as well as $\left.L_{0}\right) \operatorname{map} H_{0}^{1}(\Omega)$ onto $H^{-1}(\Omega)$, it now follows from the second estimate of $(15)$ in combination with Lemma 1, that $\left(L_{\rho_{n},\left\{z_{j}^{n}\right\}}\right)^{-1}$ converge pointwise to $L_{0}^{-1}$. As a consequence $\left(L_{\rho_{n},\left\{z_{j}^{n}\right\}}\right)^{-1} I$ converge pointwise to $L_{0}^{-1} I$.

In order to verify the collective compactness it suffices to prove that any sequence of the form

$$
\left\{\left(L_{\rho_{n_{l}},\left\{z_{j}^{n_{l}}\right\}}\right)^{-1} I u_{l}\right\}_{l=1}^{\infty}, \text { with }\left\|u_{l}\right\|_{H^{1}(\Omega)} \leq 1
$$

has a convergent subsequence. Let us first consider the case that $\rho_{n_{l}} \rightarrow 0$ as $l \rightarrow \infty$. For simplicity of notation, we shall from now on use the notation $\rho_{l}$ and $z_{j}^{l}$ in place of $\rho_{n_{l}}$ and $z_{j}^{n_{l}}$. The sequence $I u_{l}$ lies in a compact subset of $H^{-1}(\Omega)$ since $\left\|u_{l}\right\|_{H^{1}(\Omega)} \leq 1$ and since $I$ is a compact operator. $I u_{l}$ thus has a convergent subsequence, which we shall continue to index by $l$. Let $F \in H^{-1}(\Omega)$ be its limit. Then

$$
\begin{aligned}
\left\|\left(L_{\rho_{l},\left\{z_{j}^{l}\right\}}\right)^{-1} I u_{l}-L_{0}^{-1} F\right\|_{H_{0}^{1}(\Omega)} \leq & \left\|\left(L_{\rho_{l},\left\{z_{j}^{l}\right\}}\right)^{-1} I u_{l}-\left(L_{\rho_{l},\left\{z_{j}^{l}\right\}}\right)^{-1} F\right\|_{H^{1}(\Omega)} \\
& +\left\|\left(L_{\rho_{l},\left\{z_{j}^{l}\right\}}\right)^{-1} F-L_{0}^{-1} F\right\|_{H^{1}(\Omega)} \\
\leq & C_{2}\left\|I u_{l}-F\right\|_{H^{-1}(\Omega)}+\left\|\left(L_{\rho_{l},\left\{z_{j}^{l}\right\}}\right)^{-1} F-L_{0}^{-1} F\right\|_{H^{1}(\Omega)} .
\end{aligned}
$$

To get the last inequality we have used the second estimate in (15). Since the final right-hand side of the above estimate converges to zero as $l$ converges to $\infty$, it follows that the (sub)sequence $\left(L_{\rho_{l},\left\{z_{j}^{l}\right\}}\right)^{-1} I u_{l}$ is convergent.

Now consider the case that $\rho_{n_{l}}$ does not converge to 0 as $l \rightarrow \infty$. In this case there must necessarily be an index $n_{*}$, and thus a value $\rho_{*}$, and a set of points $\left\{z_{j}^{*}\right\}_{j=1}^{m}$, that are repeated infinitely often. The existence of a convergent subsequence of the sequence $\left(L_{\rho_{n_{l}},\left\{z_{j}^{n}\right\}}\right)^{-1} I u_{l}$ now follows directly from the compactness of the (fixed) operator $\left(L_{\rho_{*},\left\{z_{j}^{*}\right\}}\right)^{-1} I$. This completes the verification of the collective compactness of the original sequence $\left(L_{\rho_{n},\left\{z_{j}^{n}\right\}}\right)^{-1} I$. 
We are now ready for the:

Proof of Proposition 1. Let $s$ be subject to the lower bound (13). From our assumption (8) it follows immediately that the operator

$$
\frac{1}{s}+L_{0}^{-1} I=\frac{1}{s} L_{0}^{-1}\left(L_{0}+s\right)=\frac{\gamma^{0}}{s} L_{0}^{-1}\left(\Delta+k^{2}\right)
$$

is an isomorphism of $H_{0}^{1}(\Omega)$. Let $\rho_{n}$ be any sequence converging to 0 , and let $\left\{z_{j}^{n}\right\}_{j=1}^{m}$ be a sequence of sets of points in $\Omega$, satisfying (2). From Lemma 3 we know that the sequence $\left(L_{\rho_{n},\left\{z_{j}^{n}\right\}}\right)^{-1} I$ (and thus the sequence $\left.\left(L_{\rho_{n},\left\{z_{j}^{n}\right\}}\right)^{-1} I-L_{0}^{-1} I\right)$ is collectively compact with $\left(L_{\rho_{n},\left\{z_{j}^{n}\right\}}\right)^{-1} I \rightarrow L_{0}^{-1} I$, pointwise, as $n \rightarrow \infty$. From an application of Lemma 2 it now follows that there exist constants $N$ and $C$ such that

$$
\frac{1}{s}+\left(L_{\rho_{n},\left\{z_{j}^{n}\right\}}\right)^{-1} I \quad \text { is an isomorphism for } n \geq N
$$

and

$$
\left\|\left(\frac{1}{s}+\left(L_{\rho_{n},\left\{z_{j}^{n}\right\}}\right)^{-1} I\right)^{-1}\right\|_{\mathcal{B} \mathcal{L}\left(H_{0}^{1}(\Omega), H_{0}^{1}(\Omega)\right)} \leq C, \quad n \geq N .
$$

It now follows that the equation (17) with $\rho=\rho_{n}$ and $\left\{z_{j}\right\}_{j=1}^{m}=\left\{z_{j}^{n}\right\}_{j=1}^{m}, n \geq N$, has a unique solution for any $F \in H^{-1}(\Omega)$. Due to the equivalence of the equations (17) and (12) we conclude that (12) with $\rho=\rho_{n}$ and $\left\{z_{j}\right\}_{j=1}^{m}=\left\{z_{j}^{n}\right\}_{j=1}^{m}, n \geq N$, has a unique solution. Because of the uniform bounds (15) and (18), we furthermore conclude that this solution, $u_{n} \in H_{0}^{1}(\Omega)$, satisfies

$$
\left\|u_{n}\right\|_{H_{0}^{1}(\Omega)} \leq C\|F\|_{H^{-1}(\Omega)}
$$

with $C$ independent of $n$.

Now suppose Proposition 1 was not true. Then we could either find a sequence $\rho_{n} \rightarrow 0$ and points $\left\{z_{j}^{n}\right\}_{j=1}^{m}$, satisfying (2), such that:

(a) the problem (12), with $\rho=\rho_{n}$, and $\left\{z_{j}\right\}_{j=1}^{m}=\left\{z_{j}^{n}\right\}_{j=1}^{m}$ is not uniquely solvable (for $F \in H^{-1}(\Omega)$ ),

or we could find a sequence $\rho_{n} \rightarrow 0$ and points $\left\{z_{j}^{n}\right\}_{j=1}^{m}$, satisfying (2), such that

(b) the problem (12), with $\rho=\rho_{n}$, and $\left\{z_{j}\right\}_{j=1}^{m}=\left\{z_{j}^{n}\right\}_{j=1}^{m}$ always has a unique solution, but there exists $F_{n} \in H^{-1}(\Omega)$ such that $\quad\left\|u_{n}\right\|_{H^{1}(\Omega)}>n\left\|F_{n}\right\|_{H^{-1}(\Omega)}$.

We may without loss of generality suppose that $m$ is fixed, since according to the assumption (2) it only has a finite number of possible values. Both the situation (a) and the situation (b) represent a contradiction to what we just proved, and consequently Proposition 1 must be true. 


\section{An energy estimate}

Using the results of the preceding section we can easily derive:

Proposition 2. Suppose (2) and (8) are satisfied. Let $\rho_{0}$ be as in Proposition 1 and Corollary 1, and let $E_{\rho}$, $0<\rho<\rho_{0}$, and $E$ denote the solutions to (4)-(5) and (6)-(7) for some $f \in H^{1 / 2}(\partial \Omega)$. There exists a constant $C$, independent of $\rho$ and $f$, such that

$$
\left\|E_{\rho}-E\right\|_{L^{2}(\Omega)}+\left\|\nabla E_{\rho}-\nabla E\right\|_{L^{2}(\Omega)} \leq C \rho\|f\|_{H^{1 / 2}(\partial \Omega)}
$$

The constant $C$ depends on the domains $\left\{B_{j}\right\}_{j=1}^{m}, \Omega$, the constants $\left\{\mu^{j}, \epsilon^{j}, \sigma^{j}\right\}_{j=0}^{m}$, the frequency $\omega$, and $d_{0}$, but is otherwise independent of the points $\left\{z_{j}\right\}_{j=1}^{m}$.

Proof. The function $E_{\rho}-E$ is in $H_{0}^{1}(\Omega)$, and for any $u$ in $H_{0}^{1}(\Omega)$

$$
\begin{aligned}
\int_{\Omega}\left[\frac { 1 } { \mu _ { \rho } } \nabla \left(E_{\rho}\right.\right. & \left.-E) \cdot \nabla u-\omega^{2}\left(\epsilon_{\rho}+i \frac{\sigma_{\rho}}{\omega}\right)\left(E_{\rho}-E\right) u\right] \mathrm{d} x \\
& =\int_{\Omega}\left[-\frac{1}{\mu_{\rho}} \nabla E \cdot \nabla u+\omega^{2}\left(\epsilon_{\rho}+i \frac{\sigma_{\rho}}{\omega}\right) E u\right] \mathrm{d} x \\
& =\int_{\mathcal{I}_{\rho}}\left[\left(\frac{1}{\mu^{0}}-\frac{1}{\mu_{\rho}}\right) \nabla E \cdot \nabla u-\omega^{2}\left(\epsilon^{0}-\epsilon_{\rho}+i \frac{\sigma^{0}-\sigma_{\rho}}{\omega}\right) E u\right] \mathrm{d} x .
\end{aligned}
$$

Next

$$
\left|\int_{\mathcal{I}_{\rho}}\left[\left(\frac{1}{\mu^{0}}-\frac{1}{\mu_{\rho}}\right) \nabla E \cdot \nabla u-\omega^{2}\left(\epsilon^{0}-\epsilon_{\rho}+i \frac{\sigma^{0}-\sigma_{\rho}}{\omega}\right) E u\right] \mathrm{d} x\right| \leq C\left(\|E\|_{L^{2}\left(\mathcal{I}_{\rho}\right)}+\|\nabla E\|_{L^{2}\left(\mathcal{I}_{\rho}\right)}\right)\|u\|_{H^{1}(\Omega)} .
$$

Since $\mathcal{I}_{\rho}$ is bounded away from $\partial \Omega$, standard interior elliptic regularity results give that $\|E\|_{W^{1, \infty}\left(\mathcal{I}_{\rho}\right)} \leq$ $C\|E\|_{H^{1}(\Omega)} \leq C\|f\|_{H^{1 / 2}(\partial \Omega)}$, and so

$$
\|\nabla E\|_{L^{2}\left(\mathcal{I}_{\rho}\right)}=\left(\int_{\mathcal{I}_{\rho}}|\nabla E|^{2} \mathrm{~d} x\right)^{\frac{1}{2}} \leq\|\nabla E\|_{L^{\infty}\left(\mathcal{I}_{\rho}\right)} \rho\left(\sum_{j=1}^{m}\left|B_{j}\right|\right)^{\frac{1}{2}} \leq C \rho\|f\|_{H^{1 / 2}(\partial \Omega)},
$$

and

$$
\|E\|_{L^{2}\left(\mathcal{I}_{\rho}\right)}=\left(\int_{\mathcal{I}_{\rho}}|E|^{2} \mathrm{~d} x\right)^{\frac{1}{2}} \leq\|E\|_{L^{\infty}\left(\mathcal{I}_{\rho}\right)} \rho\left(\sum_{j=1}^{m}\left|B_{j}\right|\right)^{\frac{1}{2}} \leq C \rho\|f\|_{H^{1 / 2}(\partial \Omega)} .
$$

We conclude that $E_{\rho}-E$ satisfies

$$
\begin{aligned}
\nabla \cdot\left(\frac{1}{\mu_{\rho}} \nabla\left(E_{\rho}-E\right)\right)+\omega^{2}\left(\epsilon_{\rho}+i \frac{\sigma_{\rho}}{\omega}\right)\left(E_{\rho}-E\right) & =G \quad \text { in } \Omega, \\
E_{\rho}-E & =0 \quad \text { on } \partial \Omega,
\end{aligned}
$$

with

From Proposition 1 it then follows that

$$
\|G\|_{H^{-1}(\Omega)} \leq C \rho\|f\|_{H^{1 / 2}(\partial \Omega)} .
$$

$$
\left\|E_{\rho}-E\right\|_{H^{1}(\Omega)} \leq C \rho\|f\|_{H^{1 / 2}(\partial \Omega)},
$$

exactly as desired. 


\section{The Asymptotic Behaviour of $E_{\rho}-E$ on $\partial \mathcal{I}_{\rho}$}

For any $x \in \Omega$ let $\Phi_{0}^{k}(x, \cdot)$ denote the solution to

$$
\begin{cases}\left(\Delta+k^{2}\right) \Phi_{0}^{k}(x, \cdot)=-\delta_{x} & \text { in } \Omega \\ \Phi_{0}^{k}(x, \cdot)=0 & \text { on } \partial \Omega .\end{cases}
$$

It is not hard to see that $\Phi_{0}^{k}(x, y)=\Phi_{0}^{k}(y, x)$ for any $x, y \in \Omega($ with $x \neq y)$. For any $y \in \Omega$ we thus also have

$$
\begin{cases}\left(\Delta+k^{2}\right) \Phi_{0}^{k}(\cdot, y)=-\delta_{y} & \text { in } \Omega \\ \Phi_{0}^{k}(\cdot, y)=0 & \text { on } \partial \Omega .\end{cases}
$$

In terms of the special, free space Green's function

$$
\Phi^{k}(x, y)=\frac{i}{4} H_{0}^{(1)}(k|x-y|)
$$

introduced earlier, we have

$$
\Phi_{0}^{k}(x, y)=\Phi^{k}(x, y)+K_{1}(x, y)
$$

where $K_{1}(\cdot, \cdot)$ is in $C^{\infty}(\Omega \times \Omega)$. Furthermore $K_{1}(x, \cdot)$ is in $C^{\infty}(\bar{\Omega})$ for any $x \in \Omega$, and by symmetry, $K_{1}(\cdot, y)$ is in $C^{\infty}(\bar{\Omega})$ for any $y \in \Omega$.

It will also prove helpful to express $\Phi_{0}^{k}$ in terms of the function defined by $\Phi^{0}(x, y)=-\frac{1}{2 \pi} \log |x-y|$, a free space Green's function for the Laplacian. We have

$$
\Phi_{0}^{k}(x, y)=\Phi^{0}(x, y)+K_{2}(x, y)
$$

where $K_{2}(x, y)$ is in $C^{\infty}(\Omega \times \Omega \backslash\{(x, y): x=y\})$. For fixed $x \in \Omega$ the function $K_{2}(x, \cdot)$ satisfies

$$
\Delta K_{2}(x, \cdot)=\Delta \Phi_{0}^{k}(x, \cdot)-\Delta \Phi^{0}(x, \cdot)=-k^{2} \Phi_{0}^{k}(x, \cdot) .
$$

Due to $(20)$ and the fact that $\Phi^{k}(x, y)=-\frac{1}{2 \pi} \log |x-y|+K_{3}(x, y)$, where $K_{3}$ is uniformly bounded on any compact set (see for instance pp. 60 and 73 in [21]) it follows that $\Phi_{0}^{k}(x, \cdot)$ is in $L^{p}(\Omega)$ for any $p<\infty$. For fixed $x \in \Omega$ the function $K_{2}(x, \cdot)$ is clearly $C^{\infty}$ on $\partial \Omega$; from $(22)$ and the fact that $\Phi_{0}^{k}(x, \cdot)$ is in $L^{p}$ it now follows that

$$
K_{2}(x, \cdot) \text { is in } W^{2, p}(\Omega) \text { for any } p<\infty .
$$

The argument above also shows that the $W^{2, p}$ norm of $K_{2}(x, \cdot)$ is uniformly bounded as $x$ varies over any compact subset of $\Omega$. Sobolev's Imbedding Theorem now implies that (given a compact set $\mathcal{K} \subset \Omega$ ) there exists a constant $C$ such that

$$
\left\|K_{2}(x, \cdot)\right\|_{L^{\infty}(\Omega)}+\left\|\nabla_{y} K_{2}(x, \cdot)\right\|_{L^{\infty}(\Omega)} \leq C \text { for all } x \in \mathcal{K}
$$

In order to establish a representation formula such as that in Theorem 1, we may work on one inhomogeneity at a time, since these are a fixed minimum distance apart. In other words, we may develop representation formulas involving the difference between the electric field with $l$ inhomonegeneities and that with $l-1$ inhomogeneities, $l=m, \ldots, 1$, and then at the end essentially form the sum of these $m$ formulas (the reference field changes, but that may easily be remedied). The proof of each of the $m$ formulas is virtually identical. We only give the details when considering the difference between the electric fields corresponding to one and zero inhomogeneities. In other words, we provide the proof of Theorem 1 in the case $m=1$. In order to further simplify notation we assume that the single inhomogeneity has the form $\rho B$, that is, we assume it is "centered" at the origin (which 
we assume is contained in $\Omega$ ). The condition (2) now translates into the condition that $\operatorname{dist}(0, \partial \Omega)>d_{0}$, and we note that the remainder terms in our estimates in this and the following Section all depend on $d_{0}$, (and the shape of $B$ and $\Omega$ ) but are otherwise independent of the exact location of the origin inside $\Omega$. We denote the permeability, the permittivity and the conductivity inside $\rho B$ by $\mu^{*}, \epsilon^{*}$, and $\sigma^{*}$, respectively. Due to interior elliptic regularity results we know that $E$ is in $C^{\infty}(\Omega)$, and that $E_{\rho}$ is in $C^{0, \beta}(\Omega)$ (for some $\beta>0$ ) as well as in $C^{\infty}(\overline{\rho B})$ and in $C^{\infty}(\Omega \backslash \rho B)$ (with the normal derivative of $E_{\rho}$ having a jump across $\partial \Omega$ ). Initially we shall assume that $f$ is in $C^{1, \alpha}(\partial \Omega)$, to make sense of various boundary integrals, but this condition will be relaxed later. If $f$ is in $C^{1, \alpha}(\partial \Omega)$, then $E$ and $E_{\rho}$ are both $C^{1, \alpha}$ near and up to the boundary, $\partial \Omega$.

For $x \in \Omega \backslash \overline{\rho B}$, integration by parts provides the usual integral representation formulas for $E_{\rho}$ and $E$

$$
\begin{aligned}
E_{\rho}(x)= & \int_{\partial \Omega}\left[-E_{\rho}(y) \frac{\partial \Phi_{0}^{k}(x, y)}{\partial \nu(y)}+\frac{\partial E_{\rho}}{\partial \nu}(y) \Phi_{0}^{k}(x, y)\right] \mathrm{d} s(y) \\
& +\int_{\partial \rho B}\left[E_{\rho}(y) \frac{\partial \Phi_{0}^{k}(x, y)}{\partial \nu(y)}-\left(\frac{\partial E_{\rho}}{\partial \nu}(y)\right)^{+} \Phi_{0}^{k}(x, y)\right] \mathrm{d} s(y),
\end{aligned}
$$

and

$$
E(x)=\int_{\partial \Omega}\left[-E(y) \frac{\partial \Phi_{0}^{k}(x, y)}{\partial \nu(y)}+\frac{\partial E}{\partial \nu}(y) \Phi_{0}^{k}(x, y)\right] \mathrm{d} s(y) .
$$

Since we have chosen $\Phi_{0}^{k}$ so that $\Phi_{0}^{k}(x, y)=0$ for $x$ in $\Omega$ and $y$ on $\partial \Omega$, these representation formulas may be simplified, to read

$$
E_{\rho}(x)=-\int_{\partial \Omega} E_{\rho}(y) \frac{\partial \Phi_{0}^{k}(x, y)}{\partial \nu(y)} \mathrm{d} s(y)+\int_{\partial \rho B}\left[E_{\rho}(y) \frac{\partial \Phi_{0}^{k}(x, y)}{\partial \nu(y)}-\left(\frac{\partial E_{\rho}}{\partial \nu}(y)\right)^{+} \Phi_{0}^{k}(x, y)\right] \mathrm{d} s(y),
$$

and

$$
E(x)=-\int_{\partial \Omega} E(y) \frac{\partial \Phi_{0}^{k}(x, y)}{\partial \nu(y)} \mathrm{d} s(y) .
$$

Our first observation is:

Lemma 4. For $x$ in the open set $2 \rho B \backslash \overline{\rho B}$ :

$$
E_{\rho}(x)-E(x)=\left(1-\frac{\mu^{0}}{\mu^{*}}\right) \int_{\partial \rho B} E_{\rho}(y) \frac{\partial \Phi_{0}^{k}(x, y)}{\partial \nu(y)} \mathrm{d} s(y)+O\left(\rho^{2}|\log \rho|\right) .
$$

The term $O\left(\rho^{2}|\log \rho|\right)$ is bounded by $C \rho^{2}|\log \rho|$ uniformly in $x$. The constant $C$ depends on the shape of $B$ and $\Omega$, the constants $\mu^{*}, \epsilon^{*}, \sigma^{*}$, the constants $\mu^{0}, \epsilon^{0}, \sigma^{0}$, the frequency $\omega, d_{0}$, and the norm $\|f\|_{H^{1 / 2}(\partial \Omega)}$.

Proof. Subtracting the representation formulas (24) and (25), and recalling that $E=E_{\rho}$ on $\partial \Omega$, we get

$$
E_{\rho}(x)-E(x)=\int_{\partial \rho B}\left[E_{\rho}(y) \frac{\partial \Phi_{0}^{k}(x, y)}{\partial \nu(y)}-\left(\frac{\partial E_{\rho}}{\partial \nu}(y)\right)^{+} \Phi_{0}^{k}(x, y)\right] \mathrm{d} s(y)
$$


$x \in \Omega \backslash \overline{\rho B}$. Use of the jump condition $\frac{1}{\mu^{0}}\left(\frac{\partial E_{\rho}}{\partial \nu}(y)\right)^{+}=\frac{1}{\mu^{*}}\left(\frac{\partial E_{\rho}}{\partial \nu}(y)\right)^{-}$and the Divergence Theorem, gives

$$
\begin{aligned}
\int_{\partial \rho B}\left(\frac{\partial E_{\rho}}{\partial \nu}(y)\right)^{+} \Phi_{0}^{k}(x, y) \mathrm{d} s(y) & =\frac{\mu^{0}}{\mu^{*}} \int_{\partial \rho B}\left(\frac{\partial E_{\rho}}{\partial \nu}(y)\right)^{-} \Phi_{0}^{k}(x, y) \mathrm{d} s(y) \\
& =\frac{\mu^{0}}{\mu^{*}} \int_{\partial \rho B} E_{\rho}(y) \frac{\partial \Phi_{0}^{k}(x, y)}{\partial \nu(y)} \mathrm{d} s(y)+\left(k^{2}-\left(k^{*}\right)^{2}\right) \frac{\mu^{0}}{\mu^{*}} \int_{\rho B} E_{\rho}(y) \Phi_{0}^{k}(x, y) \mathrm{d} y,
\end{aligned}
$$

$x \in \Omega \backslash \overline{\rho B}$. Here $\left(k^{*}\right)^{2}$ denotes the constant $\left(k^{*}\right)^{2}=\omega^{2} \mu^{*}\left(\epsilon^{*}+i \frac{\sigma^{*}}{\omega}\right)$. In order to verify (26) it now suffices to show that

$$
\int_{\rho B} E_{\rho}(y) \Phi_{0}^{k}(x, y) \mathrm{d} y=O\left(\rho^{2}|\log \rho|\right), \quad x \in 2 \rho B \backslash \overline{\rho B} .
$$

This is an immediate consequence of the two estimates

$$
\begin{aligned}
\left|\int_{\rho B} E_{\rho}(y) \Phi_{0}^{k}(x, y) \mathrm{d} y\right| & \leq\left\|E_{\rho}\right\|_{L^{2}(\rho B)}\left(\int_{\rho B}\left|\Phi_{0}^{k}(x, y)\right|^{2} \mathrm{~d} y\right)^{\frac{1}{2}} \\
& \leq C\left\|E_{\rho}\right\|_{L^{2}(\rho B)}\left(\int_{0}^{C \rho} r(\log r)^{2} \mathrm{~d} r\right)^{\frac{1}{2}} \\
& \leq C\left\|E_{\rho}\right\|_{L^{2}(\rho B)} \rho|\log \rho|, \quad x \in 2 \rho B \backslash \overline{\rho B}
\end{aligned}
$$

and

$$
\begin{aligned}
\left\|E_{\rho}\right\|_{L^{2}(\rho B)} & \leq\left\|E_{\rho}-E\right\|_{L^{2}(\rho B)}+\|E\|_{L^{2}(\rho B)} \\
& \leq\left\|E_{\rho}-E\right\|_{L^{2}(\Omega)}+C \rho \\
& \leq C \rho .
\end{aligned}
$$

For the first estimate we have used (21) and (23). For the last estimate we have used Proposition 2, and the fact that $\|E\|_{L^{\infty}(\rho B)} \leq C\|E\|_{H^{1}(\Omega)} \leq C\|f\|_{H^{1 / 2}(\partial \Omega)}$. To give a shorter derivation of the estimate (27) we could alternatively have relied on the fact that $\left\|E_{\rho}\right\|_{L^{\infty}(\rho B)} \leq C\left\|E_{\rho}\right\|_{H^{1}(\Omega)} \leq C\|f\|_{H^{1 / 2}(\partial \Omega)}$; this fact follows from Theorem 8.16 of [8] and Corollary 1.

It is possible to replace $\Phi_{0}^{k}$ by the more convenient (explicit) function $\Phi^{0}(x, y)=-\frac{1}{2 \pi} \log |x-y|$.

Lemma 5. For $x$ in the open set $2 \rho B \backslash \overline{\rho B}$ :

$$
E_{\rho}(x)-E(x)=\left(1-\frac{\mu^{0}}{\mu^{*}}\right) \int_{\partial \rho B} E_{\rho}(y) \frac{\partial \Phi^{0}(x, y)}{\partial \nu(y)} \mathrm{d} s(y)+O\left(\rho^{2}|\log \rho|\right) .
$$

The term $O\left(\rho^{2}|\log \rho|\right)$ is bounded by $C \rho^{2}|\log \rho|$, uniformly in $x$. The constant $C$ depends on the shape of $B$ and $\Omega$, the constants $\mu^{*}, \epsilon^{*}, \sigma^{*}$, the constants $\mu^{0}, \epsilon^{0}, \sigma^{0}$, the frequency $\omega, d_{0}$, and the norm $\|f\|_{H^{1 / 2}(\partial \Omega)}$.

Proof. Let $\mathcal{K}$ be a compact subset of $\Omega$, containing $2 \rho B \backslash \overline{\rho B}$. As noted at the beginning of this Section

$$
\Phi_{0}^{k}(x, y)=\Phi^{0}(x, y)+K_{2}(x, y)
$$

where

$$
\left\|K_{2}(x, \cdot)\right\|_{L^{\infty}(\Omega)}+\left\|\nabla_{y} K_{2}(x, \cdot)\right\|_{L^{\infty}(\Omega)} \leq C \text { for all } x \in \mathcal{K} .
$$


The function $K_{2}(x, \cdot)$ satisfies the equation

$$
\Delta K_{2}(x, \cdot)=-k^{2} \Phi_{0}^{k}(x, \cdot) \quad \text { in } \Omega
$$

and therefore

$$
\begin{aligned}
\int_{\partial \rho B} \frac{\partial K_{2}(x, y)}{\partial \nu(y)} \mathrm{d} s(y) & =\int_{\rho B} \Delta_{y} K_{2}(x, y) \mathrm{d} y \\
& =-k^{2} \int_{\rho B} \Phi_{0}^{k}(x, y) \mathrm{d} y \\
& =O\left(\rho^{2}|\log \rho|\right), \quad x \in 2 \rho B \backslash \overline{\rho B}
\end{aligned}
$$

Using the identity (32) and the bound $\|E\|_{L^{\infty}(2 \rho B)} \leq C\|E\|_{H^{1}(\Omega)} \leq C\|f\|_{H^{1 / 2}(\partial \Omega)}$, we may now, for $x \in 2 \rho B \backslash \overline{\rho B}$, write

$$
\begin{aligned}
\int_{\partial \rho B} E_{\rho}(y) \frac{\partial K_{2}(x, y)}{\partial \nu(y)} \mathrm{d} s(y)= & \int_{\partial \rho B}\left(E_{\rho}(y)-E(x)\right) \frac{\partial K_{2}(x, y)}{\partial \nu(y)} \mathrm{d} s(y)+O\left(\rho^{2}|\log \rho|\right) \\
= & \int_{\partial \rho B}\left(E_{\rho}(y)-E(y)\right) \frac{\partial K_{2}(x, y)}{\partial \nu(y)} \mathrm{d} s(y) \\
& +\int_{\partial \rho B}(E(y)-E(x)) \frac{\partial K_{2}(x, y)}{\partial \nu(y)} \mathrm{d} s(y)+O\left(\rho^{2}|\log \rho|\right) .
\end{aligned}
$$

The individual terms in the last right-hand side may, due to (30), (31) and Proposition 2, be estimated as follows

$$
\begin{aligned}
\left|\int_{\partial \rho B}\left(E_{\rho}(y)-E(y)\right) \frac{\partial K_{2}(x, y)}{\partial \nu(y)} \mathrm{d} s(y)\right| \leq & \left|-k^{2} \int_{\rho B}\left(E_{\rho}(y)-E(y)\right) \Phi_{0}^{k}(x, y) \mathrm{d} y\right| \\
& +\left|\int_{\rho B} \nabla\left(E_{\rho}(y)-E(y)\right) \nabla_{y} K_{2}(x, y) \mathrm{d} y\right| \\
\leq & C\left\|E_{\rho}-E\right\|_{L^{2}(\rho B)}\left(\int_{\rho B}\left|\Phi_{0}^{k}(x, y)\right|^{2} \mathrm{~d} y\right)^{\frac{1}{2}} \\
& +\left\|\nabla\left(E_{\rho}-E\right)\right\| L_{L^{2}(\rho B)}\left(\int_{\rho B}\left|\nabla_{y} K_{2}(x, y)\right|^{2} \mathrm{~d} y\right)^{\frac{1}{2}} \\
\leq & C \rho^{2}|\log \rho|+C \rho^{2} \\
\leq & C \rho^{2}|\log \rho|,
\end{aligned}
$$

and

$$
\left|\int_{\partial \rho B}(E(y)-E(x)) \frac{\partial K_{2}(x, y)}{\partial \nu(y)} \mathrm{d} s(y)\right| \leq C \rho\|E(\cdot)-E(x)\|_{L^{\infty}(\partial \rho B)} \leq C \rho^{2} .
$$

Insertion of (34) and (35) into (33) yields

$$
\int_{\partial \rho B} E_{\rho}(y) \frac{\partial K_{2}(x, y)}{\partial \nu(y)} \mathrm{d} s(y)=O\left(\rho^{2}|\log \rho|\right), \quad x \in 2 \rho B \backslash \overline{\rho B} .
$$

A combination of this estimate with the decomposition (29) and Lemma 4 leads to the formula stated in the present lemma. 
Consider the limit of identity (28) as $x$ tends to $\partial \rho B$. If we invoke the well-known jump condition for double layer potentials (see [6]) we immediately get that

$$
\frac{1}{2}\left(1+\frac{\mu^{0}}{\mu^{*}}\right) E_{\rho}(x)-E(x)=\left(1-\frac{\mu^{0}}{\mu^{*}}\right) \int_{\partial \rho B} E_{\rho}(y) \frac{\partial \Phi^{0}(x, y)}{\partial \nu(y)} \mathrm{d} s(y)+O\left(\rho^{2}|\log \rho|\right),
$$

for $x$ on $\partial \rho B$. We also have that

$$
\int_{\partial \rho B} \frac{\partial \Phi^{0}(x, y)}{\partial \nu(y)} \mathrm{d} s(y)=-\frac{1}{2}
$$

for $x$ on $\partial \rho B$. Due to this last identity we may now rewrite (36) as follows

$$
\frac{1}{2}\left(1+\frac{\mu^{0}}{\mu^{*}}\right)\left(E_{\rho}(x)-E(x)\right)=\left(1-\frac{\mu^{0}}{\mu^{*}}\right) \int_{\partial \rho B}\left(E_{\rho}(y)-E(x)\right) \frac{\partial \Phi^{0}(x, y)}{\partial \nu(y)} \mathrm{d} s(y)+O\left(\rho^{2}|\log \rho|\right), \quad x \in \partial \rho B
$$

Let $\phi$ denote the vector valued function introduced earlier, i.e., the unique solution to

$$
\left\{\begin{array}{l}
\Delta \phi=0 \text { in } B, \text { and in } \mathbb{R}^{2} \backslash \bar{B} \\
\phi \text { is continuous across } \partial B \\
\frac{\mu^{*}}{\mu^{0}}\left(\frac{\partial \phi}{\partial \nu}\right)^{+}-\left(\frac{\partial \phi}{\partial \nu}\right)^{-}=-\nu \\
\lim _{|z| \rightarrow \infty}|\phi(z)|=0 .
\end{array}\right.
$$

In terms of this function we are now able to prove the following result about the asymptotic behaviour of $E_{\rho}$ on $\partial \rho B$.

Proposition 3. For $z$ on $\partial B$ :

$$
E_{\rho}(\rho z)-E(\rho z)=\rho\left(\frac{\mu^{*}}{\mu^{0}}-1\right) \phi(z) \cdot \nabla E(0)+O\left(\rho^{2}|\log \rho|\right) .
$$

The term $O\left(\rho^{2}|\log \rho|\right)$ is bounded by $C \rho^{2}|\log \rho|$, uniformly in $z$. The constant $C$ depends on the the shape of $B$ and $\Omega$, the constants $\mu^{*}, \epsilon^{*}, \sigma^{*}$, the constants $\mu^{0}, \epsilon^{0}, \sigma^{0}$, the frequency $\omega, d_{0}$, and the norm $\|f\|_{H^{1 / 2}(\partial \Omega)}$.

Proof. By introducing the formula $\frac{\partial \Phi^{0}(x, y)}{\partial \nu(y)}=-\frac{1}{2 \pi} \frac{y-x}{|y-x|^{2}} \cdot \nu(y)$ and the splitting $E_{\rho}(y)-E(x)=\left(E_{\rho}(y)-\right.$ $E(y))+(E(y)-E(x))$ into $(37)$, we obtain

$$
\begin{aligned}
\frac{1}{2}\left(1+\frac{\mu^{0}}{\mu^{*}}\right)\left(E_{\rho}(x)-E(x)\right)= & -\frac{1}{2 \pi}\left(1-\frac{\mu^{0}}{\mu^{*}}\right) \int_{\partial \rho B}\left(E_{\rho}(y)-E(y)\right) \frac{(y-x) \cdot \nu(y)}{|y-x|^{2}} \mathrm{~d} s(y) \\
& -\frac{1}{2 \pi}\left(1-\frac{\mu^{0}}{\mu^{*}}\right) \int_{\partial \rho B}(E(y)-E(x)) \frac{(y-x) \cdot \nu(y)}{|y-x|^{2}} \mathrm{~d} s(y) \\
& +O\left(\rho^{2}|\log \rho|\right), \quad x \in \partial \rho B .
\end{aligned}
$$

We now introduce $z=x / \rho$ and $\tilde{y}=y / \rho$. Since

$$
\frac{y-x}{|y-x|^{2}}=\frac{1}{\rho} \frac{\tilde{y}-z}{|\tilde{y}-z|^{2}},
$$


it follows immediately from (38) that

$$
\begin{aligned}
\frac{1}{2}\left(1+\frac{\mu^{0}}{\mu^{*}}\right)\left(E_{\rho}(\rho z)-E(\rho z)\right)= & -\frac{1}{2 \pi}\left(1-\frac{\mu^{0}}{\mu^{*}}\right) \int_{\partial B}\left(E_{\rho}(\rho \tilde{y})-E(\rho \tilde{y})\right) \frac{(\tilde{y}-z) \cdot \nu(\tilde{y})}{|\tilde{y}-z|^{2}} \mathrm{~d} s(\tilde{y}) \\
& -\frac{1}{2 \pi}\left(1-\frac{\mu^{0}}{\mu^{*}}\right) \int_{\partial B}(E(\rho \tilde{y})-E(\rho z)) \frac{(\tilde{y}-z) \cdot \nu(\tilde{y})}{|\tilde{y}-z|^{2}} \mathrm{~d} s(\tilde{y}) \\
& +O\left(\rho^{2}|\log \rho|\right), \quad z \in \partial B .
\end{aligned}
$$

Since $E$ is in $C^{2}$ in a neighborhood of $\rho B$ (with a norm that is bounded by $C\|f\|_{H^{1 / 2}(\Omega)}$ )

$$
|E(\rho \tilde{y})-E(\rho z)-\rho \nabla E(0) \cdot(\tilde{y}-z)| \leq C\left[\rho^{2}|\tilde{y}-z|^{2}+\rho^{2}|z||\tilde{y}-z|\right]
$$

and so based on (39)

$$
\begin{aligned}
\frac{1}{2}\left(1+\frac{\mu^{0}}{\mu^{*}}\right)\left(E_{\rho}(\rho z)-E(\rho z)\right)= & -\frac{1}{2 \pi}\left(1-\frac{\mu^{0}}{\mu^{*}}\right) \int_{\partial B}\left(E_{\rho}(\rho \tilde{y})-E(\rho \tilde{y})\right) \frac{(\tilde{y}-z) \cdot \nu(\tilde{y})}{|\tilde{y}-z|^{2}} \mathrm{~d} s(\tilde{y}) \\
& -\frac{\rho}{2 \pi}\left(1-\frac{\mu^{0}}{\mu^{*}}\right) \nabla E(0) \cdot \int_{\partial B}(\tilde{y}-z) \frac{(\tilde{y}-z) \cdot \nu(\tilde{y})}{|\tilde{y}-z|^{2}} \mathrm{~d} s(\tilde{y}) \\
& +O\left(\rho^{2}|\log \rho|\right), \quad z \in \partial B
\end{aligned}
$$

After insertion of the identity

$$
\begin{aligned}
-\frac{1}{2 \pi} \int_{\partial B}(\tilde{y}-z) \frac{(\tilde{y}-z) \cdot \nu(\tilde{y})}{|\tilde{y}-z|^{2}} \mathrm{~d} s(\tilde{y}) & =\int_{\partial B}(\tilde{y}-z) \frac{\Phi^{0}(z, \tilde{y})}{\partial \nu(\tilde{y})} \mathrm{d} s(\tilde{y}) \\
& =\int_{B} \nabla_{\tilde{y}}(\tilde{y}-z) \nabla_{\tilde{y}} \Phi^{0}(z, \tilde{y}) \mathrm{d} \tilde{y} \\
& =\int_{\partial B} \nu(\tilde{y}) \Phi^{0}(z, \tilde{y}) \mathrm{d} s(\tilde{y}) \\
& =-\frac{1}{2 \pi} \int_{\partial B} \nu(\tilde{y}) \log |z-\tilde{y}| \mathrm{d} s(\tilde{y}),
\end{aligned}
$$

this may be rewritten

$$
\begin{aligned}
\frac{1}{2}\left(1+\frac{\mu^{0}}{\mu^{*}}\right)\left(E_{\rho}(\rho z)-E(\rho z)\right)= & -\frac{1}{2 \pi}\left(1-\frac{\mu^{0}}{\mu^{*}}\right) \int_{\partial B}\left(E_{\rho}(\rho \tilde{y})-E(\rho \tilde{y})\right) \frac{(\tilde{y}-z) \cdot \nu(\tilde{y})}{|\tilde{y}-z|^{2}} \mathrm{~d} s(\tilde{y}) \\
& -\frac{\rho}{2 \pi}\left(1-\frac{\mu^{0}}{\mu^{*}}\right) \nabla E(0) \cdot \int_{\partial B} \nu(\tilde{y}) \log |z-\tilde{y}| \mathrm{d} s(\tilde{y}) \\
& +O\left(\rho^{2}|\log \rho|\right), \quad z \in \partial B .
\end{aligned}
$$

Fairly simple manipulations show that $\left.\phi\right|_{\partial B}$ satisfies the integral equation

$$
\begin{aligned}
& \frac{1}{2}\left(1+\frac{\mu^{0}}{\mu^{*}}\right) \phi(z)=-\frac{1}{2 \pi}\left(1-\frac{\mu^{0}}{\mu^{*}}\right) \int_{\partial B} \phi(\tilde{y}) \frac{(\tilde{y}-z) \cdot \nu(\tilde{y})}{|\tilde{y}-z|^{2}} \mathrm{~d} s(\tilde{y}) \\
& -\frac{1}{2 \pi} \frac{\mu^{0}}{\mu^{*}} \int_{\partial B} \nu(\tilde{y}) \log |z-\tilde{y}| \mathrm{d} s(\tilde{y}), \quad z \in \partial B .
\end{aligned}
$$


Indeed the uniqueness of $\phi$ guarantees that $\left.\phi\right|_{\partial B}$ is the only solution to the above equation, say, in $C^{0}(\partial B)$. Fredholm Theory now implies that the bounded linear operator $C^{0}(\partial B) \ni \psi \rightarrow(c+L)(\psi) \in C^{0}(\partial B)$, given by

$$
(c+L)(\psi)(z)=\frac{1}{2}\left(1+\frac{\mu^{0}}{\mu^{*}}\right) \psi(z)+\frac{1}{2 \pi}\left(1-\frac{\mu^{0}}{\mu^{*}}\right) \int_{\partial B} \psi(\tilde{y}) \frac{(\tilde{y}-z) \cdot \nu(\tilde{y})}{|\tilde{y}-z|^{2}} \mathrm{~d} s(\tilde{y}),
$$

maps $C^{0}(\partial B)$ onto $C^{0}(\partial B)$, and therefore has a bounded inverse.

Multiplying (41) by $\rho \frac{\mu^{*}}{\mu^{0}}\left(1-\frac{\mu^{0}}{\mu^{*}}\right) \nabla E(0)$ and subtracting it from (40), yields the following equation for $\psi^{*}(z)=E_{\rho}(\rho z)-E(\rho z)-\rho \frac{\mu^{*}}{\mu^{0}}\left(1-\frac{\mu^{0}}{\mu^{*}}\right) \nabla E(0) \cdot \phi(z)$

$$
\begin{aligned}
(c+L)\left(\psi^{*}\right)(z) & =\frac{1}{2}\left(1+\frac{\mu^{0}}{\mu^{*}}\right) \psi^{*}(z)+\frac{1}{2 \pi}\left(1-\frac{\mu^{0}}{\mu^{*}}\right) \int_{\partial B} \psi^{*}(\tilde{y}) \frac{(\tilde{y}-z) \cdot \nu(\tilde{y})}{|\tilde{y}-z|^{2}} \mathrm{~d} s(\tilde{y}) \\
& =O\left(\rho^{2}|\log \rho|\right) .
\end{aligned}
$$

Due to the existence of a bounded inverse for $c+L$ it now follows that

$$
\begin{aligned}
\left\|E_{\rho}(\rho \cdot)-E(\rho \cdot)-\rho \frac{\mu^{*}}{\mu^{0}}\left(1-\frac{\mu^{0}}{\mu^{*}}\right) \nabla E(0) \cdot \phi(\cdot)\right\|_{C^{0}(\partial B)} & =\left\|\psi^{*}\right\|_{C^{0}(\partial B)} \\
& =\left\|(c+L)^{-1} O\left(\rho^{2}|\log \rho|\right)\right\|_{C^{0}(\partial B)} \\
& \leq C \rho^{2}|\log \rho|,
\end{aligned}
$$

which is exactly the result of this lemma.

Remark. In the case of $m$ (well separated) inhomogeneities $z_{j}+\rho B_{j}, j=1, \ldots, m$, the corresponding proposition states that for $z$ on $\partial B_{j}$

$$
E_{\rho}\left(z_{j}+\rho z\right)-E\left(z_{j}+\rho z\right)=\rho\left(\frac{\mu^{j}}{\mu^{0}}-1\right) \phi_{j}(z) \cdot \nabla E\left(z_{j}\right)+O\left(\rho^{2}|\log \rho|\right) .
$$

The function $\phi_{j}$ is as defined in (9), i.e., the same as $\phi$, but with $B$ and $\mu^{*}$ replaced by $B_{j}$ and $\mu^{j}$, respectively.

\section{An asymptotic formula for $E_{\rho}-E$ a Fixed Distance AWAY From $\mathcal{I}_{\rho}$}

Just as in the previous section we derive the asymptotic formula for $E_{\rho}-E$ under the simplifying assumption, that the set of inhomogeneities $\mathcal{I}_{\rho}$ consists of just a single inhomogeneity of the form $\rho B$ (containing the origin). The material coefficients inside $\rho B$ are referred to as $\mu^{*}, \epsilon^{*}$ and $\sigma^{*}$. $\left(k^{*}\right)^{2}$ denotes the constant $\left(k^{*}\right)^{2}=\omega^{2} \mu^{*}\left(\epsilon^{*}+i \frac{\sigma^{*}}{\omega}\right)$. As before, $\Phi^{k}$ denotes the special (free space) fundamental solution for $\Delta+k^{2}$, given by

$$
\Phi^{k}(z, y)=\frac{i}{4} H_{0}^{(1)}(k|z-y|)
$$

Repeated use of Green's formula leads to the following integral representation of $E_{\rho}(x)-E(x)$, for points $x \in \Omega \backslash \overline{\rho B}$

$$
\begin{aligned}
E_{\rho}(x)-E(x)= & \int_{\partial \Omega}\left(\frac{\partial E_{\rho}}{\partial \nu}(y)-\frac{\partial E}{\partial \nu}(y)\right) \Phi^{k}(x, y) \mathrm{d} s(y) \\
& +\int_{\partial \rho B}\left[E_{\rho}(y) \frac{\partial \Phi^{k}(x, y)}{\partial \nu(y)}-\left(\frac{\partial E_{\rho}}{\partial \nu}(y)\right)^{+} \Phi^{k}(x, y)\right] \mathrm{d} s(y) .
\end{aligned}
$$


Using the jump condition $\left(\frac{\partial E_{\rho}}{\partial \nu}(y)\right)^{+}=\frac{\mu^{0}}{\mu^{*}}\left(\frac{\partial E_{\rho}}{\partial \nu}(y)\right)^{-}, y \in \partial \rho B$, we calculate

$$
\begin{aligned}
\int_{\partial \rho B}\left(\frac{\partial E_{\rho}}{\partial \nu}(y)\right)^{+} \Phi^{k}(x, y) \mathrm{d} s(y) & =\frac{\mu^{0}}{\mu^{*}} \int_{\partial \rho B}\left(\frac{\partial E_{\rho}(y)}{\partial \nu(y)}\right)^{-} \Phi^{k}(x, y) \mathrm{d} s(y) \\
& =\frac{\mu^{0}}{\mu^{*}} \int_{\partial \rho B} E_{\rho}(y) \frac{\partial \Phi^{k}(x, y)}{\partial \nu(y)} \mathrm{d} s(y)+\left(k^{2}-\left(k^{*}\right)^{2}\right) \frac{\mu^{0}}{\mu^{*}} \int_{\rho B} E_{\rho}(y) \Phi^{k}(x, y) \mathrm{d} y
\end{aligned}
$$

The representation (42) may thus be rewritten

$$
\begin{aligned}
E_{\rho}(x)-E(x)= & \int_{\partial \Omega}\left(\frac{\partial E_{\rho}}{\partial \nu}(y)-\frac{\partial E}{\partial \nu}(y)\right) \Phi^{k}(x, y) \mathrm{d} s(y)+\left(1-\frac{\mu^{0}}{\mu^{*}}\right) \int_{\partial \rho B} E_{\rho}(y) \frac{\partial \Phi^{k}(x, y)}{\partial \nu(y)} \mathrm{d} s(y) \\
& -\left(k^{2}-\left(k^{*}\right)^{2}\right) \frac{\mu^{0}}{\mu^{*}} \int_{\rho B} E_{\rho}(y) \Phi^{k}(x, y) \mathrm{d} s(y)
\end{aligned}
$$

$x \in \Omega \backslash \overline{\rho B}$. Proposition 3 from the previous section now allows us to find the exact asymptotic behaviour of the last two terms of this representation.

Lemma 6. For any fixed $x \in \mathbb{R}^{2} \backslash \overline{\rho B}$ we have

$$
\int_{\rho B} E_{\rho}(y) \Phi^{k}(x, y) \mathrm{d} s(y)=\rho^{2} \Phi^{k}(x, 0)|B| E(0)+O\left(\rho^{3-\delta}\right),
$$

and

$$
\int_{\partial \rho B} E_{\rho}(y) \frac{\partial \Phi^{k}(x, y)}{\partial \nu(y)} \mathrm{d} s(y)=\rho^{2} \nabla_{y} \Phi^{k}(x, 0) \cdot M\left(\frac{\mu^{*}}{\mu^{0}}\right) \nabla E(0)-\rho^{2} k^{2} \Phi^{k}(x, 0)|B| E(0)+O\left(\rho^{3-\delta}\right) .
$$

The polarization tensor is as defined previously, i.e.,

$$
M\left(\frac{\mu^{*}}{\mu^{0}}\right)=|B| I+\left(\frac{\mu^{*}}{\mu^{0}}-1\right) \int_{\partial B} \nu(y)(\phi(y))^{T} \mathrm{~d} s(y) .
$$

Given any fixed $\delta>0$, and any fixed compact subset $\mathcal{K} \subset \mathbb{R}^{2} \backslash \overline{\rho B}$, with $\operatorname{dist}(\mathcal{K}, 0) \geq d_{0} / 2$, there exists constants $C$ such that the remainder terms, as well as their derivatives, are bounded by $C \rho^{3-\delta}$, uniformly with respect to $x \in \mathcal{K}$. The constants $C$ depend on $\delta, \mathcal{K}$, the shape of $B$ and $\Omega$, the constants $\mu^{*}, \epsilon^{*}, \sigma^{*}$, the constants $\mu^{0}, \epsilon^{0}, \sigma^{0}$, the frequency $\omega, d_{0}$, and the norm $\|f\|_{H^{1 / 2}(\partial \Omega)}$.

Proof. Since $\Omega$ is a bounded, smooth domain in $\mathbb{R}^{2}$, Sobolev's Imbedding Theorem gives

$$
\left\|E_{\rho}-E\right\|_{L^{p}(\Omega)} \leq C\left\|E_{\rho}-E\right\|_{H^{1}(\Omega)} \text { for any } \quad 1<p<\infty,
$$

where $C$ depends on $\Omega$ and $p$ only. Proposition 2 therefore asserts that

$$
\left\|E_{\rho}-E\right\|_{L^{p}(\Omega)} \leq C \rho .
$$

This immediately leads to the estimate

$$
\left|\int_{\rho B}\left(E_{\rho}(y)-E(y)\right) \Phi^{k}(x, y) \mathrm{d} y\right| \leq C\left\|E_{\rho}-E\right\|_{L^{p}(\Omega)}\left(\rho^{2}\right)^{\frac{1}{p^{\prime}}} \leq C \rho\left(\rho^{2}\right)^{\frac{1}{p^{\prime}}}
$$


for any fixed $x \in \mathbb{R}^{2} \backslash \overline{\rho B}$. We may choose $p=\frac{2}{\delta}\left(\frac{1}{p^{\prime}}=1-\frac{\delta}{2}\right)$, in which case

$$
\left|\int_{\rho B}\left(E_{\rho}(y)-E(y)\right) \Phi^{k}(x, y) \mathrm{d} y\right| \leq C \rho^{3-\delta} .
$$

Due to the interior smoothness of $E(\cdot)$, and the fact that $x$ is fixed and outside $\overline{\rho B}$

$$
\int_{\rho B} E(y) \Phi^{k}(x, y) \mathrm{d} y=\rho^{2}|B| E(0) \Phi^{k}(x, 0)+O\left(\rho^{3}\right) .
$$

A combination of (44) and (45) yields

$$
\int_{\rho B} E_{\rho}(y) \Phi^{k}(x, y) \mathrm{d} y=\rho^{2}|B| E(0) \Phi^{k}(x, 0)+O\left(\rho^{3-\delta}\right),
$$

the first of the identities in this lemma. It is easy to see that the remainder $O\left(\rho^{3-\delta}\right)(x)$ is bounded by $C \rho^{3-\delta}$, uniformly on the compact subset $\mathcal{K}$ (which satisfies $\left.\operatorname{dist}(\mathcal{K}, 0) \geq d_{0} / 2\right)$. Since $O\left(\rho^{3-\delta}\right)(x)$ clearly satisfies $\left(\Delta+k^{2}\right) O\left(\rho^{3-\delta}\right)=0$ in $\mathbb{R}^{2} \backslash \overline{\rho B}$, and since $O\left(\rho^{3-\delta}\right)(x)$ is bounded by $C \rho^{3-\delta}$, uniformly on (any) $\mathcal{K}$, standard interior elliptic estimates immediately show, that similar bounds hold for all the derivatives of $O\left(\rho^{3-\delta}\right)(x)$.

We now turn our attention to the second identity. From the Divergence Theorem

$$
\int_{\partial \rho B} E(y) \frac{\partial \Phi^{k}(x, y)}{\partial \nu(y)} \mathrm{d} s(y)=\int_{\rho B} \nabla E(y) \nabla_{y} \Phi^{k}(x, y) \mathrm{d} s(y)-k^{2} \int_{\rho B} E(y) \Phi^{k}(x, y) \mathrm{d} s(y),
$$

for $x \in \mathbb{R}^{2} \backslash \overline{\rho B}$. Since $E$ is in $C^{2}$ in a fixed neighborhood of $\rho B$, and since $x$ is fixed and outside $\overline{\rho B}$, this immediately yields

$$
\int_{\partial \rho B} E(y) \frac{\partial \Phi^{k}(x, y)}{\partial \nu(y)} \mathrm{d} s(y)=\rho^{2} \nabla_{y} \Phi^{k}(x, 0) \cdot|B| \nabla E(0)-\rho^{2} k^{2} \Phi^{k}(x, 0)|B| E(0)+O\left(\rho^{3}\right) .
$$

As a consequence we have

$$
\begin{aligned}
\int_{\partial \rho B} E_{\rho}(y) \frac{\partial \Phi^{k}(x, y)}{\partial \nu(y)} \mathrm{d} s(y)= & \int_{\partial \rho B}\left(E_{\rho}(y)-E(y)\right) \frac{\partial \Phi^{k}(x, y)}{\partial \nu(y)} \mathrm{d} s(y)+\int_{\partial \rho B} E(y) \frac{\partial \Phi^{k}(x, y)}{\partial \nu(y)} \mathrm{d} s(y) \\
= & \int_{\partial \rho B}\left(E_{\rho}(y)-E(y)\right) \frac{\partial \Phi^{k}(x, y)}{\partial \nu(y)} \mathrm{d} s(y)+\rho^{2} \nabla_{y} \Phi^{k}(x, 0) \cdot|B| \nabla E(0) \\
& -\rho^{2} k^{2} \Phi^{k}(x, 0)|B| E(0)+O\left(\rho^{3}\right) .
\end{aligned}
$$

From Proposition 3 we get

$$
\begin{aligned}
\int_{\partial \rho B}\left(E_{\rho}(y)-E(y)\right) \frac{\partial \Phi^{k}(x, y)}{\partial \nu(y)} \mathrm{d} s(y)= & \rho \int_{\partial B}\left(E_{\rho}(\rho \tilde{y})-E(\rho \tilde{y})\right) \nabla_{y} \Phi^{k}(x, \rho \tilde{y}) \cdot \nu(\tilde{y}) \mathrm{d} s(\tilde{y}) \\
= & \rho^{2} \int_{\partial B}\left(\frac{\mu^{*}}{\mu^{0}}-1\right) \phi(\tilde{y}) \cdot \nabla E(0) \nabla_{y} \Phi^{k}(x, \rho \tilde{y}) \cdot \nu(\tilde{y}) \mathrm{d} s(\tilde{y}) \\
& +\rho \int_{\partial B} O\left(\rho^{2}|\log \rho|\right) \nabla_{y} \Phi^{k}(x, \rho \tilde{y}) \cdot \nu(\tilde{y}) \mathrm{d} s(\tilde{y}) \\
= & \rho^{2} \int_{\partial B}\left(\frac{\mu^{*}}{\mu^{0}}-1\right) \phi(\tilde{y}) \cdot \nabla E(0) \nabla_{y} \Phi^{k}(x, \rho \tilde{y}) \cdot \nu(\tilde{y}) \mathrm{d} s(\tilde{y}) \\
& +O\left(\rho^{3}|\log \rho|\right),
\end{aligned}
$$


for any fixed $x \in \mathbb{R}^{2} \backslash \overline{\rho B}$. For such a point $x$ we also have

$$
\nabla_{y} \Phi^{k}(x, \rho \tilde{y})=\nabla_{y} \Phi^{k}(x, 0)+O(\rho), \quad \tilde{y} \in \partial B
$$

and (47) thus gives

$$
\int_{\partial \rho B}\left(E_{\rho}(y)-E(y)\right) \frac{\partial \Phi^{k}(x, y)}{\partial \nu(y)} \mathrm{d} s(y)=\rho^{2}\left(\frac{\mu^{*}}{\mu^{0}}-1\right) \nabla_{y} \Phi^{k}(x, 0) \cdot\left\{\int_{\partial B} \nu(y)(\phi(y))^{T} \mathrm{~d} s(y)\right\} \nabla E(0)+O\left(\rho^{3-\delta}\right)
$$

for any fixed $\delta>0$. This verifies the second identity of this lemma. It is easy to see that the remainder $O\left(\rho^{3-\delta}\right)(x)$ is bounded by $C \rho^{3-\delta}$, uniformly on the compact subset $\mathcal{K}$, (which $\left.\operatorname{satisfies} \operatorname{dist}(\mathcal{K}, 0) \geq d_{0} / 2\right)$. The bounds for the derivatives of the term $O\left(\rho^{3-\delta}\right)$ follow by the same (interior elliptic estimate) argument as before.

Proof of Theorem 1. An application of Lemma 6 to (43) leads to the following asymptotic representation formula

$$
\begin{aligned}
E_{\rho}(x)-E(x)= & \int_{\partial \Omega}\left(\frac{\partial E_{\rho}}{\partial \nu}(y)-\frac{\partial E}{\partial \nu}(y)\right) \Phi^{k}(x, y) \mathrm{d} s(y)+\rho^{2}\left(1-\frac{\mu^{0}}{\mu^{*}}\right) \nabla_{y} \Phi^{k}(x, 0) \cdot M\left(\frac{\mu^{*}}{\mu^{0}}\right) \nabla E(0) \\
& -\rho^{2} \omega^{2} \mu^{0}\left(\epsilon^{0}-\epsilon^{*}+i \frac{\sigma^{0}-\sigma^{*}}{\omega}\right) \Phi^{k}(x, 0)|B| E(0)+O\left(\rho^{3-\delta}\right),
\end{aligned}
$$

for any fixed $x \in \Omega \backslash \overline{\rho B}$. We note that the term $O\left(\rho^{3-\delta}\right)$, and all its derivatives, are bounded by $C \rho^{3-\delta}$, uniformly with respect to $x$ satisfying $\operatorname{dist}(x, \partial \Omega) \leq d_{0} / 2$. We may extend the outward normal field to $\partial \Omega$ to a small neighbourhood inside $\Omega$ and apply the operator $\frac{\partial}{\partial \nu(x)}$ to both sides of (48), the result being

$$
\begin{aligned}
\frac{\partial E_{\rho}}{\partial \nu(x)}(x)-\frac{\partial E}{\partial \nu(x)}(x)= & \int_{\partial \Omega}\left(\frac{\partial E_{\rho}}{\partial \nu}(y)-\frac{\partial E}{\partial \nu}(y)\right) \frac{\partial \Phi^{k}(x, y)}{\partial \nu(x)} \mathrm{d} s(y)+\rho^{2}\left(1-\frac{\mu^{0}}{\mu^{*}}\right) \nabla_{y} \frac{\partial \Phi^{k}}{\partial \nu(x)}(x, 0) \cdot M\left(\frac{\mu^{*}}{\mu^{0}}\right) \nabla E(0) \\
& -\rho^{2} \omega^{2} \mu^{0}\left(\epsilon^{0}-\epsilon^{*}+i \frac{\sigma^{0}-\sigma^{*}}{\omega}\right) \frac{\partial \Phi^{k}(x, 0)}{\partial \nu(x)}|B| E(0)+O\left(\rho^{3-\delta}\right)
\end{aligned}
$$

By means of an argument identical to that used for $\Phi_{0}^{k}$, it is not difficult to show that

$$
\Phi^{k}(x, y)=\Phi^{0}(x, y)+K_{3}(x, y)
$$

where $K_{3}$ is in $C^{\infty}\left(\mathbb{R}^{2} \times \mathbb{R}^{2} \backslash\{(x, y): x=y\}\right)$, and $K_{3}$ furthermore satisfies the estimate

$$
\|K(\cdot, \cdot)\|_{L^{\infty}(\mathcal{K} \times \mathcal{K})}+\left\|\nabla_{x} K(\cdot, \cdot)\right\|_{L^{\infty}(\mathcal{K} \times \mathcal{K})}+\left\|\nabla_{y} K(\cdot, \cdot)\right\|_{L^{\infty}(\mathcal{K} \times \mathcal{K})} \leq C_{\mathcal{K}}
$$

for any compact set $\mathcal{K} \subset \mathbb{R}^{2}$. From these properties of $K_{3}$ it follows immediately that boundary integrals involving the potential $\frac{\partial \Phi^{k}(x, y)}{\partial \nu(x)}$ exhibit the same jump relations as those involving $\frac{\partial \Phi^{0}(x, y)}{\partial \nu(x)}=-\frac{1}{2 \pi} \frac{(x-y) \cdot \nu(x)}{|x-y|^{2}}$ (the normal derivative of a standard single layer potential). For more details on this point we refer the reader to [6], page 47 . In particular, by letting $x$ tend to a limiting point on $\partial \Omega$, we obtain from (49)

$$
\begin{array}{r}
\frac{1}{2}\left(\frac{\partial E_{\rho}}{\partial \nu}(x)-\frac{\partial E}{\partial \nu}(x)\right)-\int_{\partial \Omega}\left(\frac{\partial E_{\rho}}{\partial \nu}(y)-\frac{\partial E}{\partial \nu}(y)\right) \\
=\rho^{2}\left(1-\frac{\mu^{0}}{\mu^{*}}\right) \nabla_{y} \frac{\partial \Phi^{k}}{\partial \nu(x)}(x, 0) \cdot M\left(\frac{\mu^{*}}{\mu^{0}}\right) \nabla E(0) \\
\quad-\rho^{2} \omega^{2} \mu^{0}\left(\epsilon^{0}-\epsilon^{*}+i \frac{\sigma^{0}-\sigma^{*}}{\omega}\right) \frac{\partial \Phi^{k}(x, 0)}{\partial \nu(x)}|B| E(0)+O\left(\rho^{3-\delta}\right)
\end{array}
$$


for any $x \in \partial \Omega$. For any fixed $\delta$ the term $O\left(\rho^{3-\delta}\right)$ is bounded by $C \rho^{3-\delta}$ uniformly in $x$. The constant $C$ depends on $\delta$, the shape of $B$ and $\Omega$, the constants $\mu^{*}, \epsilon^{*}, \sigma^{*}$, the constants $\mu^{0}, \epsilon^{0}, \sigma^{0}$, the frequency $\omega, d_{0}$, and the norm $\|f\|_{H^{1 / 2}(\partial \Omega)}$. So far we have assumed that $f$ is in $C^{1, \alpha}(\partial \Omega)$ to insure that $\frac{\partial E_{\rho}}{\partial \nu}$ and $\frac{\partial E}{\partial \nu}$ are continuous functions on $\partial \Omega$. We notice, however, that even if $f$ is only in $H^{1 / 2}(\partial \Omega)$, the difference $E_{\rho}-E$ is infinitely smooth near and up to $\partial \Omega$ (this follows from elliptic regularity results, and the fact that $E_{\rho}-E$ vanishes on $\partial \Omega$ ). The difference $\frac{\partial E_{\rho}}{\partial \nu}-\frac{\partial E}{\partial \nu}$ is therefore also infinitely smooth near and up to $\partial \Omega$. By a limiting argument (using the density of $C^{1, \alpha}(\partial \Omega)$ in $H^{1 / 2}(\partial \Omega)$ ) it easily follows that the last formula remains valid under the (weaker) condition that $f$ is in $H^{1 / 2}(\partial \Omega)$. This verifies Theorem 1 in the case of one inhomogeneity. As already mentioned earlier, the general case is verified essentially by an iteration of this argument. We leave the details to the reader.

Remark. We could equally well start with fixed Neuman boundary conditions for $E_{\rho}$ and $E$, say $\frac{1}{\mu^{0}} \frac{\partial}{\partial \nu} E_{\rho}=$ $\frac{1}{\mu^{0}} \frac{\partial}{\partial \nu} E=g$ on $\partial \Omega$. The goal is then to derive an asymptotic representation formula involving $\left.\left(E_{\rho}-E\right)\right|_{\partial \Omega}$. Instead of (42) we now have

$$
\begin{aligned}
E_{\rho}(x)-E(x)= & -\int_{\partial \Omega}\left(E_{\rho}(y)-E(y)\right) \frac{\partial \Phi^{k}(x, y)}{\partial \nu(y)} \mathrm{d} s(y) \\
& +\int_{\partial \rho B}\left[E_{\rho}(y) \frac{\partial \Phi^{k}(x, y)}{\partial \nu(y)}-\left(\frac{\partial E_{\rho}}{\partial \nu}(y)\right)^{+} \Phi^{k}(x, y)\right] \mathrm{d} s(y) .
\end{aligned}
$$

As long as the boundary conditions for $E_{\rho}$ and $E$ are fixed, and identical, it does not matter whether they be Dirichlet or Neumann, as far as our asymptotic analysis of the behaviour near the inhomogeneities is concerned. That is to say, the preceding analysis (from Sects. 4 to 6) immediately carries over; at the appropriate places we just replace the Dirichlet-fundamental solution $\Phi_{0}^{k}$ with the corresponding Neumann-fundamental solution $\tilde{\Phi}_{0}^{k}$, defined by

$$
\begin{cases}\left(\Delta+k^{2}\right) \tilde{\Phi}_{0}^{k}(x, \cdot)=-\delta_{x} & \text { in } \Omega \\ \frac{\partial}{\partial \nu} \Phi(x, \cdot)=0 & \text { on } \partial \Omega\end{cases}
$$

Remember, in this case we suppose $-k^{2}$ is not an eigenvalue for the Laplacian with homogeneous Neumann boundary conditions. From (50) we now arrive at

$$
\begin{aligned}
E_{\rho}(x)-E(x)= & -\int_{\partial \Omega}\left(E_{\rho}(y)-E(y)\right) \frac{\partial \Phi^{k}(x, y)}{\partial \nu(y)} \mathrm{d} s(y)+\rho^{2}\left(1-\frac{\mu^{0}}{\mu^{*}}\right) \nabla_{y} \Phi^{k}(x, 0) \cdot M\left(\frac{\mu^{*}}{\mu^{0}}\right) \nabla E(0) \\
& -\rho^{2} \omega^{2} \mu^{0}\left(\epsilon^{0}-\epsilon^{*}+i \frac{\sigma^{0}-\sigma^{*}}{\omega}\right) \Phi^{k}(x, 0)|B| E(0)+O\left(\rho^{3-\delta}\right),
\end{aligned}
$$

for any fixed $x$ in $\Omega \backslash \overline{\rho B}$. As before, we may take the limit of this representation as $x$ converges to a point on $\partial \Omega$, to finally get

$$
\begin{aligned}
& \begin{aligned}
\frac{1}{2}\left(E_{\rho}(x)-E(x)\right)+\int_{\partial \Omega}\left(E_{\rho}(y)-E(y)\right) \frac{\partial \Phi^{k}(x, y)}{\partial \nu(y)} & \mathrm{d} s(y) \\
=\rho^{2}\left(1-\frac{\mu^{0}}{\mu^{*}}\right) & \nabla_{y} \Phi(x, 0) \cdot M\left(\frac{\mu^{*}}{\mu^{0}}\right) \nabla E(0) \\
& -\rho^{2} \omega^{2} \mu^{0}\left(\epsilon^{0}-\epsilon^{*}+i \frac{\sigma^{0}-\sigma^{*}}{\omega}\right) \Phi^{k}(x, 0)|B| E(0)+O\left(\rho^{3-\delta}\right),
\end{aligned}
\end{aligned}
$$

$x \in \partial \Omega$. This provides a proof of the representation in Theorem 2, for the case of one inhomogeneity. The proof for any fixed number of well separated inhomogeneities follows (as before) essentially by iteration of the 
argument just presented. The statements about the remainder term $O\left(\rho^{3-\delta}\right)$ are as in Theorem 1 , except that the norm $\|f\|_{H^{1 / 2}(\partial \Omega)}$ is replaced by the norm $\|g\|_{H^{-1 / 2}(\partial \Omega)}$.

\section{Discussion}

As mentioned earlier, we expect that the asymptotic formulas of Theorem 1 (or Theorem 2) will serve as very useful tools for the (numerical) reconstruction of the "location" and "size" of the inhomogeneities. If for instance the electric field, $E_{\rho}$ is prescribed on $\partial \Omega(=f)$, and the rescaled magnetic field $\frac{\partial E_{\rho}}{\partial \nu}$ is measured on $\partial \Omega$, then the function

$$
\frac{\partial E_{\rho}}{\partial \nu}(x)-\frac{\partial E}{\partial \nu}(x)-2 \int_{\partial \Omega}\left(\frac{\partial E_{\rho}}{\partial \nu}-\frac{\partial E}{\partial \nu}\right)(y) \frac{\partial \Phi^{k}(x, y)}{\partial \nu(x)} \mathrm{d} s(y)
$$

may be considered a measured datum on $\partial \Omega$ (the constants $\mu^{0}, \epsilon^{0}$, and $\sigma^{0}$ are assumed to be known, and we may easily compute $E$ ). From the asymptotic formula in Theorem 1 it now follows that, up to terms of smaller order, we are in possession of the values of the (boundary) function

$$
\begin{aligned}
2 \rho^{2} \sum_{j=1}^{m} & \left(1-\frac{\mu^{0}}{\mu^{j}}\right) \nabla_{y} \frac{\partial \Phi^{k}}{\partial \nu(x)}\left(x, z_{j}\right) \cdot M_{j}\left(\frac{\mu^{j}}{\mu^{0}}\right) \nabla E\left(z_{j}\right) \\
& -2 \rho^{2} \omega^{2} \mu^{0} \sum_{j=1}^{m}\left(\epsilon^{0}-\epsilon^{j}+i \frac{\sigma^{0}-\sigma^{j}}{\omega}\right) \frac{\partial \Phi^{k}}{\partial \nu(x)}\left(x, z_{j}\right)\left|B_{j}\right| E\left(z_{j}\right) .
\end{aligned}
$$

A first task of the identification process, is then to determine (as well as possible) the number of "poles" ("centers" of inhomogeneities) $z_{j}$, and their locations. A second task would be to determine other information about the inhomogeneities, such as their sizes, and/or other "geometric" and "parametric" information. Disregarding the magnitude of the involved constants, the formula (51) suggests that inhomogeneities with permittivity or conductivity different from that of the background will be easier to "locate" based on TE electromagnetic boundary data, than will inhomogeneities where only the permeability differ (the decay away from $x=z_{j}$ is more rapid for the first term than for the second). TM electromagnetic data reverse this situation. We are indeed currently in the process of experimenting with numerical reconstructions. To assess the practical usefulness of our approach, we use values for the piecewise constant coefficients that are characteristic of actual materials, such as soil, metals and plastics, and we use frequencies that are characteristic of fairly standard radar devices. A detailed account of this work will be the subject of a forthcoming paper.

We have based our asymptotic formulas on the free space Green's function $\Phi^{k}$, given by the formula

$$
\Phi^{k}(x, y)=\frac{i}{4} H_{0}^{(1)}(k|x-y|) \text {. }
$$

As the reader will notice, we have never used the particular properties of this function at $\infty$ (it satisfies the so-called Sommerfeld radiation condition) and indeed, we might just as well have used any other free space Green's function for the operator $\Delta+k^{2}$. If anything, our reason for choosing $\Phi^{k}(x, y)$ is its status as somewhat of a "standard" Green's function for the operator $\Delta+k^{2}$. We also note that due to the knowledge of a rapidly convergent series representation for $H_{0}^{(1)}$, the function $\Phi^{k}(x, y)=\frac{i}{4} H_{0}^{(1)}(k|x-y|)$ is in a sense just as explicit as say the Green's function $\Phi^{0}(x, y)=-\frac{1}{2 \pi} \log |x-y|$ for the Laplacian.

The formula from Theorem 2 would be used when the boundary magnetic field $\frac{1}{\mu^{0}} \frac{\partial \tilde{E}}{\partial \nu}$ is prescribed, and the boundary electric field $\left.\tilde{E}\right|_{\partial \Omega}$ is measured. In practice it may be unnatural to distinguish between measured and prescribed data, since in a sense they will both be measured. It would then be natural to consider the datum, which is measured most accurately, as the prescribed datum, use this as the basis for the calculation of the "background" field ( $E$ or $\tilde{E})$ and then use the corresponding expression (from Th. 1 or Th. 2) for the 
reconstruction process. We note that the expression from Theorem 2 only involves one derivative of $\Phi^{k}-$ if anything, this may make it slightly simpler to compute with.

Acknowledgements. This research was partially supported by NSF grant DMS-9704575.

\section{REFERENCES}

[1] H. Ammari, S. Moskow and M. Vogelius, Boundary integral formulas for the reconstruction of electromagnetic imperfections of small diameter. Preprint, Rutgers University (1999); Inverse Problems (submitted).

[2] P.M. Anselone, Collectively Compact Operator Approximation Theory and Applications to Integral Equations. Prentice-Hall, Englewood Cliffs, New Jersey (1971).

[3] L. Baratchart, J. Leblond, F. Mandréa and E.B. Saff, How can meromorhic approximation help to solve some 2D inverse problems for the Laplacian? Inverse Problems 15 (1999) 79-90.

[4] J. Blitz, Electrical and Magnetic Methods of Nondestructive Testing. IOP Publishing, Adam Hilger, New York (1991).

[5] D. Cedio-Fengya, S. Moskow and M.S. Vogelius, Identification of conductivity imperfections of small diameter by boundary measurements. Continuous dependence and computational reconstruction. Inverse Problems 14 (1998) 553-595.

[6] D. Colton and R. Kress, Integral Equation Methods in Scattering Theory. Krieger Publishing Co., Malabar, Florida (1992).

[7] D. Dobson and F. Santosa, Nondestructive evaluation of plates using eddy current methods. Internat. J. Engrg. Sci. 36 (1998) 395-409.

[8] D. Gilbarg and N.S. Trudinger, Elliptic Partial Differential Equations of Second Order, 2nd Ed., Springer-Verlag, New York (1983).

[9] D. Griffiths, Introduction to Electrodynamics, 2nd Ed., Prentice Hall, Upper Saddle River, New Jersey (1989).

[10] F. Gylys-Colwell, An inverse problem for the Helmholtz equation. Inverse Problems 12 (1996) 139-156.

[11] J.D. Jackson, Classical Electrodynamics, 2nd Ed., Wiley, New York (1975).

[12] R. Kohn and M. Vogelius, Determining conductivity by boundary measurements. Comm. Pure Appl. Math. 37 (1984) $289-298$. II. Interior results. Comm. Pure Appl. Math. 38 (1985) 643-667.

[13] M. Lassas, The impedance imaging problem as a low-frequency limit. Inverse Problems 13 (1997) 1503-1518.

[14] N.N. Lebedev, Special Functions \& 3 Their Applications. Dover Publications, New York (1972).

[15] A. Nachman, Global uniqueness for a two-dimensional inverse boundary value problem. Ann. of Math. 143 (1996) 71-96.

[16] P. Ola, L. Päivärinta and E. Somersalo, An inverse boundary value problem in electrodynamics. Duke Math. J. 70 (1993) 617-653.

[17] A. Sahin and E.L. Miller, Electromagnetic scattering-based array processing methods for near-field object characterization. Preprint, Northeastern University (1998).

[18] E. Somersalo, D. Isaacson and M. Cheney, A linearized inverse boundary value problem for Maxwell's equations. J. Comput. Appl. Math. 42 (1992) 123-136.

[19] J. Sylvester and G. Uhlmann, A uniqueness theorem for an inverse boundary value problem in electrical prospection. Comm. Pure Appl. Math. 39 (1986) 91-112.

[20] J. Sylvester and G. Uhlmann, A global uniqueness theorem for an inverse boundary value problem. Ann. of Math. 125 (1987) 153-169.

[21] G.N. Watson, A Treatise on the Theory of Bessel Functions, 2nd Ed., Cambridge University Press, London (1962).

To access this journal online:

www.edpsciences.org 